\title{
Non-measurable automorphisms of Lie groups relative to the real- and non-archimedean-valued measures
}

\author{
S.V. Ludkovsky
}

15.10.2007

\begin{abstract}
In this work the problem about an existence of non-measurable automorphisms of Lie groups finite and as well infinite dimensional over the field of real numbers and also over the non-archimedean local fields is investigated. Non-measurability of automorphisms is considered relative to real-valued measures and also measures with values in non-archimedean local fields. Their existence is proved and a procedure for their construction is given. Their application for a construction of non-measurable irreducible unitary representations is demonstrated.
\end{abstract}

\section{Introduction}

Continuous automorphisms of Lie groups were widely studied [4, 18, 23, 21], but discontinuous and non-measurable automorphisms are known substantially less [2, 13, 7, 8, 9]. In this work an explicit direct procedure of a construction of non-measurable automorphisms of locally compact Lie groups is given and it is proved that their existence is a local property. Moreover, non-measurable automorphisms of locally compact Lie algebras are constructed. Their application for the construction of weakly non-measurable irreducible unitary representations of locally compact groups is given. In this article basic necessary facts are reminded. Besides this non-measurable automorphisms of infinite dimensional over the real field, as well as non-archimedean fields of zero characteristic, Lie groups, which are not locally compact are studied. Non-measurable automorphisms on definite more general topological groups are investigated. The basic results of the second section of the paper are Theorems 13, 15, 16, 19, 20 and Corollaries 14, 17, while in Corollary 14, Theorem 19 and $\S 13$ the specific features of Lie groups are taken into account.

Besides real-valued measures here in the third section apart from the previous works nonmeasurability of automorphisms of totally disconnected topological groups is investigated also for measures with values in infinite locally compact fields of zero characteristic with non-archimedean non-trivial multiplicative norms, that is with values in local fields.

\section{Non-measurable automorphisms of groups relative to real-valued measures}

1. Definitions. For groups $G$ and $S$ a mapping $f: G \rightarrow S$ is called a homomorphism, if it preserves the multiplication operation, that is $f(a b)=f(a) f(b)$ for each $a, b \in G$. If a homomorphism $f$ is bijective and surjective from $G$ onto $S, f(G)=S$, then the homomorphism 
$f$ is called the (algebraic) isomorphism. In the case $G=S$ an isomorphism $f$ is called the automorphism.

A homomorphism $f: G \rightarrow U(H)$ is called a unitary representation of the group $G$, if $H$ is the Hilbert or the unitary space over $\mathbf{C}, U(H)$ is the unitary group of $H$. In the particular case $H=\mathbf{C}$, that is $U(\mathbf{C})=S^{1}=\{z \in \mathbf{C}:|z|=1\}$, a homomorphism $f: G \rightarrow S^{1}$ is called a character of the group $G$.

If $\mathrm{g}$ is a Lie algebra over the field $\mathbf{F}$, then a bijective surjective mapping $\phi: \mathrm{g} \rightarrow \mathrm{g}$ we call an automorphism, if it preserves addition and multiplication: $\phi(a+b)=\phi(a)+\phi(b)$, $\phi([a, b])=[\phi(a), \phi(b)]$ for each $a, b \in \mathrm{g}$.

A topological space $X$ is called compact, if from each its open covering there is possible to extract a finite subcovering. A topological space $X$ is called locally compact, if each its point $x \in X$ has a neighborhood $U$, the closure of which $\bar{U}$ is compact (see [25]; in [18] the old topological terminology is slightly different from the new one [25]).

For a locally compact Hausdorff topological group $G$ a non-negative non-trivial $\sigma$-additive measure $\mu$ on the $\sigma$-algebra $\mathcal{B}(G)$ of all Borel subsets in $G$ is called a left (or right) Haar measure, if $\mu(g A)=\mu(A)$ (or $\mu(A g)=\mu(A)$ respectively) for each $A \in \mathcal{B}(G)$ and $g \in G$.

Let a $\sigma$-algebra $\mathcal{A}(G)=\mathcal{A}_{\mu}(G)$ be a completion of the Borel $\sigma$-algebra $\mathcal{B}(G)$ with the help of subsets $P$ in $G$ such that $P \subset F \in \mathcal{B}(G)$ and $\mu(F)=0$, where $\mu$ is a non-negative non-trivial $\sigma$-additive measure on the $\sigma$-algebra $\mathcal{B}(G)$. An automorphism $f$ of a topological group $G$ is called $\mu$-measurable, if $f^{-1}(U) \in \mathcal{A}(G)$ for each $U \in \mathcal{B}(G)$. In the contrary case an automorphism is called $\mu$-non-measurable.

A set $X$ with a $\sigma$-algebra of its subsets $\mathcal{U}$ is called a measurable space and it is denoted by $(X, \mathcal{U})$.

A measure $\nu$ is called absolutely continuous relative to the measure $\mu$ on the measurable space $(X, \mathcal{U})$, if from $\mu(A)=0$ it follows $\nu(A)=0$, where $A \in \mathcal{U}$. Measures $\mu$ and $\nu$ are called equivalent, if they are absolutely continuous relative to each other.

2. Lemma. Let $G$ be a separable locally compact non-compact group, and let $\mu$ be a non-negative Haar measure on $G$. Then on the one-point compactification $\alpha G$ (considered as the topological space) there exists a finite measure $\nu$ equivalent to the measure $\mu$.

Proof. For each locally compact non-compact topological space their exists its one-point (Alexandroff) compactification due to Theorem 3.5.11 [25]. Take an open neighborhood $U$ of the unit element in $G$ such that $\mu(U)<\infty$. Since $\mu$ is non-trivial, then $\mu(U)>0$. Due to the separability of the group $G$ there exists a countable family $\left\{g_{j}: j \in \mathbf{N}\right\}$ of elements in $G$ such that $\bigcup_{j} g_{j} U=G$, where $g U=\{z: z=g f, f \in U\}$. Put

$$
\nu(A):=\sum_{j=1}^{\infty} \mu\left(\left(g_{j} U\right) \cap A\right) / 2^{j}(1)
$$

for each $A \in \mathcal{A}(G)$ and $\nu(\{\alpha\})=0$, where $\{\alpha\}=\alpha G \backslash G$ is the compactification enlargement. Then the measure $\nu$ is defined on $\mathcal{A}(\alpha G)$ and $0<\nu(\alpha G)<\infty$. In view of Formula (1) $\nu(A)=0$ if and only if $\mu(A)=0$. Therefore, measures $\mu$ and $\nu$ are equivalent.

3. Lemma. If $\mu$ and $\nu$ are two equivalent $\sigma$-additive measures on $\mathcal{B}(G)$, where $G$ is a topological group, then an automorphism $f$ is $\mu$-measurable if and only if it is $\nu$-measurable.

Proof. Since measures $\mu$ and $\nu$ are equivalent and on the same $\sigma$-algebra $\mathcal{B}(G)$ are given, then $\mathcal{A}_{\mu}(G)=\mathcal{A}_{\nu}(G)$. From the definition of measurability of the automorphism the statement of this lemma follows.

4. Definitions. A subgroup $H$ of a group $G$ is called normal, if its left and right cosets coincide $g H=H g$ for each $g \in G$. A group $G$ is called algebraically simple, if it has not a normal subgroup different from $e$ and $G$, where $e=e_{G}$ is the unit element of the group $G$. A topological group $G$ is called topologically simple, if it has not a normal closed subgroup different from $e$ and $G$. 
5. Lemma. If $f: G \rightarrow V$ is a homomorphism of an algebraically simple group $G$ into a group $V$, then either $f^{-1}\left(e_{V}\right)=e_{G}$ or $f^{-1}\left(e_{V}\right)=G$.

Proof. The subgroup $J=f^{-1}\left(e_{V}\right)$ is normal in $G$, since if $f(g)=e_{V}$, then $f\left(h^{-1} g h\right)=$ $f^{-1}(h) f(g) f(h)=f^{-1}(h) f(h)=f\left(h^{-1} h\right)=f\left(e_{G}\right)=e_{V}$ for each $h \in G$. Due to the definition of the algebraic simplicity of the group $G$ either $J=e_{G}$ or $J=G$.

6. Corollary. If an algebraically simple group $G$ has a character $f: G \rightarrow S^{1}$, then either $f^{-1}(1)=e_{G}$ or $f^{-1}(1)=G$, where $S^{1}:=\{z \in \mathbf{C}:|z|=1\}$ is the multiplicative Abelian group.

7. Remark. If $f^{-1}\left(e_{V}\right)=e_{G}$ for a homomorphism $f: G \rightarrow V$, then $f$ is bijective. In this case $f(G)$ is (algebraically) isomorphic with $G$. If moreover $V$ is Abelian, for example, $V=S^{1}$, then $G$ is Abelian and can not be simple, besides the trivial case $G=e$.

8. Lemma. If a Hausdorff topological group $G$ is topologically simple and $N$ is a normal subgroup in $G$, then either $N=e_{G}$ or $\bar{N}=G$, where $\bar{N}=c_{G} N$ is the closure of $N$ in $G$.

Proof. Each topological group is the uniform space with the entourages of the diagonal of the form $W(U):=\left\{(g, q) \in G \times G: g^{-1} q \in U\right\}$, where $U$ is an open neighborhood of $e$ in $G$ (see Example 8.1.17 in [25]). If $g \in \bar{N}$, then there exists a net $\left\{g_{\alpha}: \alpha \in \Lambda\right\}$ such that $\lim g_{\alpha}=g$, where $\Lambda$ is a directed set, $g_{\alpha} \in N$ for each $\alpha \in \Lambda$ (see $\S 1.6$ and Corollary 8.1.4 in [25]). Since $h^{-1} g_{\alpha} h \in N$ for each $h \in G$, then due to the continuity of the multiplication in $G$ there is satisfied the equality $\lim h^{-1} g_{\alpha} h=h^{-1} g h$, hence $h^{-1} \bar{N} h=\bar{N}$ for each $h \in G$. Then $\bar{N}$ is the closed normal subgroup in $G$. In view of the topological simplicity of the group $G$ either $\bar{N}=e$ or $\bar{N}=G$. Since $N \subset \bar{N}$, then in the case of $\bar{N}=e$ we get $N=e$.

9. Corollary. If $f: G \rightarrow V$ is a continuous homomorphism of a topologically simple group $G$ in a topological group $V$, where $G$ and $V$ are Hausdorff, then either $f^{-1}\left(e_{V}\right)=e_{G}$ or $f^{-1}\left(e_{V}\right)=G$.

Proof. In a Hausdorff topological space each singleton is closed (see $\S 1.5$ in [25]). Since the homomorphism $f$ is continuous, then $f^{-1}\left(e_{V}\right)=: N$ is closed in $G$. On the other hand, $N$ is the normal subgroup in $G$. From Lemma 8 this Corollary follows.

10. Remark. Henceforth, Lie groups $G$ of the smoothness class $C^{\infty}$ over the field of real numbers or $C^{\omega}$ over local fields, that is finite algebraic extension of the field of $p$-adic numbers, are considered, where a smoothness class is for $G$ as the manifold and for the smoothness of the operation $G \times G \ni(g, q) \mapsto g^{-1} q \in G, C^{\infty}$ denotes the class of infinitely differentiable mappings, $C^{\omega}$ denotes the class of locally analytic mappings. As usually $U(n)$ denotes the unitary group of the unitary space $\mathbf{C}^{\mathbf{n}}$, where $n$ is the natural number.

11. Lemma. If $G$ is a locally compact Lie group over $\mathbf{R}$ of the dimension $n$, then there exists an open neighborhood $U$ of the unit element e in $G$, which has a topological embedding into $\left(S^{1}\right)^{n}$, as well as an embedding into $U(n)$ as the local Lie group.

Proof. For a locally compact Lie group $G$ of the smoothness class $C^{\infty}$ over $\mathbf{R}$ as it is well-known there exists a Lie algebra $\mathrm{g}=T_{e} G$ and an exponential mapping exp : $V_{1} \rightarrow U_{1}$ of an open neighborhood $V_{1}$ of zero in $g$ on an open neighborhood $U_{1}$ of the unit element $e$ in $G$, while exp is the infinite differentiable diffeomorphism, $U_{1}$ is a local Lie group (see [4, 12, 18]).

As the linear space over $\mathbf{R}$ the algebra $\mathrm{g}$ has the dimension $n$, consequently, there exists the embedding of $U_{1}$ into $\mathbf{R}^{\mathbf{n}}$. Choose a compact subset $V$ in $V_{1}$, such that the interior $\operatorname{Int}(V)$ is the open neighborhood of zero in $\mathrm{g}$. Then $\exp (V)=: U$ is the compact subset in $U_{1}$, moreover, $\exp (\operatorname{Int}(V))$ is the local subgroup in $G$. Therefore, $U$ has a topological embedding into $\mathbf{R}^{\mathbf{n}} / \mathbf{Z}^{\mathbf{n}}$, where the latter topological space is isomorphic with $\left(S^{1}\right)^{n}$. Since the Lie algebra $\mathrm{g}$ has the basis of generators $v_{1}, \ldots, v_{m}$ of a dimension not exceeding $n$, then each element in $U$ can be presented as the finite product of local one-parameter subgroups $\exp \left(t_{j} v_{j}\right)$ with $t_{j} \in(-\epsilon, \epsilon)$, where $\epsilon>0$. For a sufficiently small $\epsilon>0$ each $\left\{\exp \left(t_{j} v_{j}\right): t_{j} \in(-\epsilon, \epsilon)\right\}$ has an 
embedding into $S^{1}$ as the Abelian local subgroup.

In $\mathrm{g}$ in the open neighborhood of zero there is accomplished the Campbell-Hausdorff formula (see Chapter III in [4]).

Remind that the Campbell-Hausdorff formula for the calculation of the expression $w=$ $\ln \left(e^{u} e^{v}\right)$ in the neighborhood of zero $V$ of the Lie algebra $g$ over $\mathbf{K}=\mathbf{R}$ or a local nonarchimedean field $\mathbf{K}$ has the form:

$$
\begin{aligned}
& w=\sum_{n=1}^{\infty} n^{-1} \sum_{r+s=n, r \geq 0, s \geq 0}\left(\tilde{w}_{r, s}+\hat{w}_{r, s}\right), \text { where } \\
& \tilde{w}_{r, s}:=\sum_{m \geq 1}(-1)^{m+1} m^{-1} \sum^{*}\left(\left(\prod_{i=1}^{m-1}(a d u)^{r_{i}}\left(r_{i} !\right)^{-1}(\text { ad } v)^{s_{i}}\left(s_{i} !\right)^{-1}\right)\right. \\
& \left.(a d u)^{r_{m}}\left(r_{m} !\right)^{-1}\right)(v), \\
& \hat{w}_{r, s}:=\sum_{m \geq 1}(-1)^{m+1} m^{-1} \sum^{* *}\left(\prod_{i=1}^{m-1}(a d u)^{r_{i}}\left(r_{i} !\right)^{-1}(a d v)^{s_{i}}\left(s_{i} !\right)^{-1}\right)(u),
\end{aligned}
$$

where $\sum^{*}$ denotes the sum by $r_{1}+\ldots+r_{m}=r, s_{1}+\ldots+s_{m-1}=s-1, r_{1}+s_{1} \geq 1, \ldots, r_{m-1}+$ $s_{m-1} \geq 1$, while $\sum^{* *}$ denotes the sum by $r_{1}+\ldots+r_{m-1}=r-1, s_{1}+\ldots+s_{m-1}=s$, $r_{1}+s_{1} \geq 1, \ldots, r_{m-1}+s_{m-1} \geq 1$,

where the convergence radius of the series depends on $\mathbf{K}$ and the multiplicative norm in it.

Each local one-parameter subgroup $\exp (t v)$ with $t \in(-\epsilon, \epsilon)$ acts on $\mathrm{g}$ and then has the embedding into $G l(n, \mathbf{R})$, therefore, $U$ has the embedding into $G L(n, \mathbf{R})$ (see also Theorems 58, 59, 84, 87-90 and Propositions 42(A,B), 56(A,B,C) in Chapter $10 \S \S 42,53,56$ and 57 [18).

The Lie algebra $\mathrm{u}(n)$ has the basis of generators $E_{k, j}-E_{j, k}, i\left(E_{k, j}+E_{j, k}\right)$ with $1 \leq$ $k<j \leq n$ and $i E_{j, j}$ with $j=1, \ldots, n$, where $i=(-1)^{1 / 2}, E_{k, j}$ is the $n \times n$ matrix with 1 on the crossing of the $k$-th row and $j$-th column and others elements are zero. The Lie algebra $\mathrm{g}$ has the embedding into $\mathrm{gl}(n, \mathbf{R})$, while each generator of the algebra $\mathrm{gl}(n, \mathbf{R})$ is the linear combination of generators of the algebra $\mathrm{u}(n)$, hence $\mathrm{g}$ has the embedding into $\mathrm{u}(n)$ as the Lie subalgebra. Then $\exp _{u} \circ \ln _{G}: U_{1} \rightarrow U(n)$ gives the embedding, where $\exp _{u}$ is the exponential mapping for the Lie algebra $\mathrm{u}(n)$ of the Lie group $U(n), \ln _{G}$ is the logarithmic mapping for $G$ from $U$ into $V$.

Certainly, in general an embedding of a local Lie subgroup may have not an extension over the entire group.

12. Lemma. Let $X$ be a Tychonoff (completely regular) dense in itself topological space with $\sigma$-additive, $\sigma$-finite non-negative Borel regular measure $\mu$ on a complete $\sigma$-algebra $\mathcal{A}_{\mu}$ such that for each $x \in X$ there exists an open neighborhood $U$ of a finite positive measure $0<\mu(U)<\infty$, moreover, $\mu$ has not atoms in $X$. If $f: X \rightarrow X$ is the bijective epimorphic mapping such that card $(f(U) \cap V) \geq c:=\operatorname{card}(\mathbf{R})$ for each open subsets $U$ and $V$ in $X$, then $f$ and $f^{-1}$ are not $\left(\mathcal{A}_{\mu}, \mathcal{B}\right)$-measurable.

Proof. Recall, that a measure $\mu$ is called Borel, if it is defined on the $\sigma$-algebra of all Borel subsets $\mathcal{B}(X)$ in $X$, that is the minimal $\sigma$-algebra generated by the family of all open subsets in $X$. In the given case the algebra $\mathcal{A}_{\mu}$ is the minimal $\sigma$-algebra, produced from $\mathcal{B}(X)$ and the family of all subsets of $\mu$ measure zero. A measure $\mu$ is called Borel regular, if $\mu(A)=\sup \{\mu(C): C \subset A, C$ is closed $\}$ for each $A \in \mathcal{B}(X)$. If $\mu(A)<\infty$, then the transition to the completion gives $\mu(A)=\inf \{\mu(V): A \subset V$ open $\}$, since the measure $\mu$ is $\sigma$-finite (see Theorem 2.2.2 [22]).

Let $U$ and $V$ be open in $X$, while by the condition of the Lemma $\operatorname{card}(f(U) \cap V) \geq \mathrm{c}$, then $f^{-1}(f(U) \cap V)=U \cap f^{-1}(V)$, since $f$ is the bijective mapping from $X$ onto $X$, consequently, $\operatorname{card}\left(U \cap f^{-1}(V) \geq \mathrm{c}\right.$ for each $U$ and $V$ open in $X$. If $A \in \mathcal{A}_{\mu}, \mu(A)<\infty$, then there exists a Borel subset $B \in \mathcal{B}(X)$ such that $A \subset B$ and $\mu(A)=\mu(B)$. Then for each $\epsilon>0$ there exists an open subset $W$ in $X$ such that $A \subset W$ and $\mu(A) \leq \mu(W)<\mu(A)+\epsilon$. If $S$ is an arbitrary subset in $X$, then by $\mu^{*}(S)$ there is denoted $\inf \{\mu(W): S \subset W, W$ is open $\}=: \mu^{*}(S)$.

For arbitrary open subsets $U$ and $V$ of a finite $\mu$ measure take open subsets $U_{1}$ and $U_{2}$ 
in $U, V_{1}$ and $V_{2}$ in $V$ such that $0<\mu(U) / 2-\delta<\mu\left(U_{j}\right)<\mu(U) / 2+\delta$ and $0<\mu(V) / 2-\delta<$ $\mu\left(V_{j}\right)<\mu(V) / 2+\delta$ for $j=1,2$, where $0<\delta<\min (\mu(U), \mu(V)) / 9$. This is possible, since $\mu$ is non-negative, has not any atom, while for each $x \in X$ there exists an open subset $P$ with $x \in P, 0<\mu(P)<\infty$. Denote $A:=f^{-1}(U), A_{j}:=f^{-1}\left(U_{j}\right)$. By the supposition of this lemma $\operatorname{card}\left(A_{j} \cap V_{k}\right) \geq \mathrm{c}$ for each $j, k \in\{1,2\}$. Suppose that $f$ is a $\left(\mathcal{A}_{\mu}, \mathcal{B}(X)\right)$ measurable mapping. Then there would be $A_{j} \in \mathcal{A}_{\mu}$ for $j=1,2$ and there would exist $B_{j} \in \mathcal{B}(X)$ such that $A_{j} \subset B_{j}$ and $\mu\left(B_{j}\right)=A_{j}$, and hence open subsets would be $W_{j}$ with $B_{j} \subset W_{j}$ and $\mu\left(B_{j}\right) \leq \mu\left(W_{j}\right)<\mu\left(B_{j}\right)+\delta$ for $j=1,2$. But $A_{1} \cap A_{2}=\emptyset$, consequently, $\mu(A)=\mu\left(A_{1}\right)+\mu\left(A_{2}\right)$.

If for all open $U$ there would be $\mu\left(f^{-1}(U) \cap V\right)=0$ for each open $V$ in $X$ with $\mu(V)<$ $\infty$, then in view of the $\sigma$-finiteness and $\sigma$-additivity of $\mu$ then would be $\mu(X)=0$, that contradicts the supposition of this lemma, therefore, there can be chosen open subsets $U, U_{1}$ and $U_{2}$ such that $\mu\left(A_{j} \cap V\right)>0$, where $U_{1} \cup U_{2} \subset U$. But $\operatorname{card}\left(A_{j} \cap P_{k}\right) \geq \mathrm{c}$ for each $P_{k}$ open subset in $V_{k}$.

On the other hand, $\mu^{*}\left(A_{j} \cap V_{k}\right)=\inf \left\{\mu(Y): Y\right.$ is open, $\left.Y \supset\left(A_{j} \cap V_{k}\right)\right\}>0$, consequently, there exists a countable sequence of open sets $Y_{n} \supset\left(A_{j} \cap V_{k}\right)$ such that $\lim _{n \rightarrow \infty} \mu\left(Y_{n}\right)=$ $\mu^{*}\left(A_{j} \cap V_{k}\right)$. Let $C_{n}=\bigcap_{s=1}^{n} Y_{s}$, then $\left(A_{j} \cap V_{k}\right) \subset C_{n+l} \subset C_{n}$ for every $n, l \in \mathbf{N}$, each $C_{n}$ is open. Moreover, $\lim _{n \rightarrow \infty} \mu\left(C_{n}\right)=\mu^{*}\left(A_{j} \cap V_{k}\right)$. Since $\operatorname{card}\left(A_{j} \cap P\right) \geq \mathrm{c}$ for each $P$ open in $X$, then $\operatorname{Int}\left(C_{n} \backslash C_{n+l}\right)=\emptyset$ for all $n, l \in \mathbf{N}$, where $\operatorname{Int}(B)$ denotes the interior of a subset $B$ in $X$. Then $\mu^{*}\left(A_{j} \cap V_{k}\right)=\mu^{*}\left(\left[c l_{X}\left(A_{j} \cap V_{k}\right)\right] \cap V_{k}\right)=\mu\left(\left[c l_{X}\left(A_{j} \cap V_{k}\right)\right] \cap V_{k}\right)$, since $c l_{X}\left(A_{j} \cap V_{k}\right) \in \mathcal{B}(X)$, while $X$ is the completely regular space dense in itself, where $c l_{X}(B)$ denotes the closure of a subset $B$ in $X$. Thus, $\mu^{*}\left(A_{j} \cap V_{k}\right) \geq \mu(V) / 2-\delta$, since $c l_{X}\left(A_{j} \cap V_{k}\right)=c l_{X}\left(V_{k}\right) \supset V_{k}$. Therefore, $\mu\left(A_{1} \cap V_{1}\right)+\mu\left(A_{1} \cap V_{2}\right)+\mu\left(A_{2} \cap V_{1}\right)+\mu\left(A_{2} \cap V_{2}\right) \geq 2 \mu(V)-8 \delta>10 \mu(V) / 9$ and the contradiction is obtained, since by the construction $A_{1} \cap A_{2}=\emptyset, V_{1} \cap V_{2}=\emptyset$ and $V_{1} \cup V_{2} \subset V$, consequently, $f$ is not measurable.

Applying the above proof to $f^{-1}$ instead of $f$ we get, that $f^{-1}$ is also nonmeasurable, since $f^{-1}$ satisfies conditions from the second section of the proof.

13. Theorem. Let $G$ be a non-trivial locally compact Lie group over $\mathbf{R}$ or over a non-archimedean local field $\mathbf{K}$. Then the group of its automorphisms Aut $(G)$ has a family of the cardinality not less than $2^{\mathrm{c}}$ of different non-measurable automorphisms relative to a non-trivial non-negative Haar measure $\mu$ on $G$, where $c:=\operatorname{card}(\mathbf{R})$ denotes the cardinality of the continuum.

Proof. Each locally compact Lie group $G$ has a finite dimensional Lie algebra g over $\mathbf{K}=\mathbf{R}$ or over a non-archimedean local field $\mathbf{K}$ respectively. Therefore, both $G$ and $\mathbf{g}$ are metrizable, moreover the metric can be chosen left-invariant (see Theorem 8.3 [23]).

In the non-archimedean case as a neighborhood $U$ of the unit element $e$ in $G$ there can be chosen a compact clopen subgroup, since $G$ is totally disconnected (see Theorems 5.13 and 7.7 [23]). If $\mathbf{K}=\mathbf{R}$, then we take an open symmetric $U=U^{-1}$ neighborhood $U$ of the unit element $e$ in $G$, where $U^{-1}:=\left\{g^{-1}: g \in U\right\}$. For a sufficiently small $U$ there is a bijective exponential mapping exp : $V \rightarrow U$ from the corresponding neighborhood $V$ of zero in $\mathrm{g}$ on $U$, where exp belongs to the class of smoothness $C^{\infty}$ or $C^{\omega}$ (see [4, 15, 12, 18]). Choose $U$ sufficiently small, that in it would be satisfied the Campbell-Hausdorff formula.

Take a basis of generators $v_{1}, \ldots, v_{m}$ in the Lie algebra $\mathrm{g}$ over the field $\mathbf{K}$, and as the linear space over the field $\mathbf{K}$ it has the basis $\eta_{1}, \ldots, \eta_{n}$, where $n \geq m, v_{j}=\eta_{j}$ for $j=1, \ldots, m$, and $\eta_{m+1}, \ldots, \eta_{n}$ are obtained as finite products (commutators) $[u, v]$ of basic generators in the Lie algebra $\mathrm{g}$ for $n>m$.

Consider a set $\mathbf{K} \backslash \mathbf{Q}$ of all irrational elements of the field $\mathbf{K}$. The field $\mathbf{K}$ is uncountable and as the linear space over $\mathbf{Q}$ it is infinite-dimensional, since the field of rational numbers $\mathbf{Q}$ is countable. For each $b \in \mathbf{K} \backslash \mathbf{Q}$ there exists an extension $\mathbf{Q}(b)$ of the field $\mathbf{Q}$ with the 
help of a number $b, \mathbf{Q} \subset \mathbf{Q}(b)$. Remind that a number $a \in \mathbf{K} \backslash \mathbf{F}$ is called algebraic over the field $\mathbf{F}$, if it is a root of a polynomial with coefficients from $\mathbf{F}$. If an element $a$ is not algebraic over $\mathbf{F}$, then it is called transcendental. For a transcendental element $a$ over a field $\mathbf{F}$ the field of rational quotients with coefficients from the field $\mathbf{F}$ is purely transcendental.

A set $\left\{a_{1}, \ldots, a_{n}\right\}$ from $\mathbf{K}$ is algebraically independent, if each polynomial $P\left(x_{1}, \ldots, x_{n}\right)$ with coefficients from $\mathbf{F}$ becoming zero while substitution of $a_{1}, \ldots, a_{n}$ instead of $x_{1}, \ldots, x_{n}$, is the identically zero polynomial. Moreover, the field $\left(\mathbf{F}\left(a_{1}, \ldots, a_{n-1}\right)\right)\left(a_{n}\right)$ is isomorphic with the field of rational quotients $\mathbf{F}\left(a_{1}, \ldots, a_{n}\right)$ of variables $a_{1}, \ldots, a_{n}$ with coefficients from the field $\mathbf{F}$. If a field $\mathbf{F}$ is countable, then $\mathbf{F}\left(a_{j}: j \in \mathbf{N}\right)$ is also countable, since $\bigcup_{n=1}^{\infty} \aleph_{0}^{n}=\aleph_{0}$, where $\aleph_{0}=\operatorname{card}(\mathbf{N}), \mathbf{N}$ denotes the set of natural numbers [25].

A subset (not necessarily finite) $S$ in $\mathbf{K} \backslash \mathbf{F}$ is called algebraically independent over $\mathbf{F}$, if each its finite subset is algebraically independent. In the family of all algebraically independent subsets in $\mathbf{K}$ over $\mathbf{F}$ there exists a partial ordering by the inclusion, that makes it directed. In view of the Kuratowski-Zorn lemma there exists a maximal relative to such ordering algebraically independent family $\Psi$ in $\mathbf{K}$ over $\mathbf{F}$. Each such subset is called the basis of transcendence of the field $\mathbf{K}$ over the field $\mathbf{F}$. In view of Theorem of section 1.1.5 [1] the cardinal numbers of any transcendence bases coincide.

Since by the G. Cantor theorem a set $A$ of all algebraic numbers over a countable field is countable, hence $\operatorname{card}(\Psi)=\mathrm{c}=\operatorname{card}(\mathbf{R})$, in particular, for $\mathbf{F}=\mathbf{Q}$. Moreover, the Haar measure $\nu$ of algebraic numbers in $\mathbf{K}$ is equal to zero, $\nu(A)=0$, where $\nu$ is non-negative and non-trivial on $\mathbf{K}$. Then $\mathbf{Q} \subset \mathbf{Q}(\Psi) \subset \mathbf{K}$, where $\mathbf{F}(\Psi)$ denotes the purely transcendental extension of the field $\mathbf{F}$, while $\mathbf{Q}(\Psi) \subset \mathbf{K}$ is the algebraic extension (see section 1.1.5 [1]).

The Lie algebra $\mathrm{g}$ is infinite-dimensional over $\mathbf{Q}$ with an uncountable Hamel basis $\gamma$ over $\mathbf{Q}$, that is $w_{1}, \ldots, w_{k}$ are linearly independent over the field of rational numbers $\mathbf{Q}$ for each $w_{1}, \ldots, w_{k} \in \gamma$ and $k \in \mathbf{N}$, while each element $w \in \mathrm{g}$ is the finite linear combination $w=c_{1} w_{1}+\ldots+c_{k} w_{k}$ over the field $\mathbf{Q}$ of elements $w_{j}$ from $\gamma$ with rational coefficients $c_{j} \in \mathbf{Q}$.

In the non-archimedean case $U$ is the subgroup in $G$ (see above), then put $W=U$. In the case of the Lie group $G$ over the field of real numbers $\mathbf{R}$ the neighborhood $U$ generates the group $W:=\bigcup_{n=1}^{\infty} U^{n}$ containing in it the connected component $C$ of the unit $e$ (see Theorem $7.4[23]$ ), where $A B:=\{g h: g \in A, h \in B\}$ for $A, B \subset G$.

Let $g \in G$ be an element of the group $G$, then elements of the form $g^{n}$ for $n \in \mathbf{Z}$ generate the commutative subgroup $S(g)$ in $G$. If a subgroup $S(g)$ is finite, then its order is called the order $\operatorname{ord}(g)=k$ of the element $g$, that is $S(g)=\left\{e, g, g^{2}, \ldots, g^{k-1}\right\}$ and $g^{k}=e$. If $S(g)$ is infinite, then it is said, that $g$ is the element of the infinite order. Let $G_{f i n}:=\operatorname{gr}(\mathcal{F})$ be a minimal subgroup in $G$, generated by all possible finite products of elements from $\mathcal{F}$, where $\mathcal{F}$ is the set of all elements of finite orders in $G$. Then we consider the subgroup $W_{\text {fin }}=G_{f i n} \cap W$ in $G$. Let $U_{\text {fin }}=W_{\text {fin }} \cap U$. Then $\ln \left(U_{\text {fin }}\right) \subset \mathrm{g}$, where $\ln : U \rightarrow V$ is the logarithmic mapping of a local Lie subgroup on a neighborhood of zero in the Lie algebra g, corresponding to the Campbell-Hausdorff formula, where coefficients of the series are rational numbers (see $\S 11$ ). Consider the minimal subalgebra $g_{\text {fin }}$ over the field of rational numbers $\mathbf{Q}$ generated by $\ln \left(U_{\text {fin }}\right)$ and $v_{1}, \ldots, v_{m}, \mathrm{~g}_{\text {fin }} \subset \mathrm{g}$, since $\mathbf{Q} \subset \mathbf{K}$.

Consider one-parameter local subgroups. Each element from the open neighborhood $U$ of the unit element belongs to a local one-parameter subgroup $\left\{g^{t}: t \in \mathbf{K},|t|<\epsilon\right\}=g_{\text {loc }}$, where $\epsilon>0$, which is unique for each non-unit element $g \neq e$ from $U$. Each cyclic commutative group is isomorphic to the group of roots of 1 in C. Therefore, on each subgroup $g_{W}:=$ $\bigcup_{n=1}^{\infty}\left(g_{l o c}\right)^{n}$ the set of elements of finite orders has the Haar measure $\tilde{\mu}_{g}\left(g_{W}\right)=0$ zero, where $\tilde{\mu}_{g}$ is the Haar measure on $g_{W}$. Then $\mu\left(W_{f i n}\right)=0$ and $\nu^{n}\left(g_{\text {fin }}\right)=0$, since the image $\mu_{l n}$ of the measure $\mu$ on $V$ is equivalent to $\left.\nu^{n}\right|_{V}$, where $\nu^{n}$ is the Haar measure on $\mathbf{K}^{\mathbf{n}}$ as the additive group, $\mu_{l n}(B)=\mu(\exp (B))$ for each $B \in \mathcal{B}(V)$. 
If $g \in G, \operatorname{ord}(g)=k \in \mathbf{N}$ and $h^{m}=g, m \in \mathbf{N}$, then $\operatorname{ord}(h) \leq m k \in \mathbf{N}$. If $g \in U, g=e^{v}$, $v \in V, \operatorname{ord}(g)=k \in \mathbf{N}, h=e^{t v} \in U$ for some $t \in \mathbf{K}, \operatorname{ord}(h) \in \mathbf{N}$, then $t \in \mathbf{Q}$.

Let $\mathbf{Q}(A)$ denotes the minimal field, containing all elements from $A$ and such that $\mathbf{Q} \subset$ $\mathbf{Q}(A) \subset \mathbf{K}$. Among subsets $A \subset \Psi$ take such that $\mathrm{g}(A) \supset \mathrm{g}_{\text {fin }}$, where $\mathrm{g}(A)$ is the Lie algebra over the field $\mathbf{Q}(A)$ with the Hamel basis $\gamma_{A}$ as the $\mathbf{Q}(A)$-linear space, $\gamma_{A} \subset \gamma, \gamma$ denotes the Hamel basis $\mathrm{g}$ over $\mathbf{Q}$ as the $\mathbf{Q}$-linear subspace (see above). Moreover, it is possible to restrict the consideration by $A$ such that $\left(\nu^{n}\right)^{*}(\mathrm{~g}(A))=0$, since $\nu^{n}\left(\mathrm{~g}_{\text {fin }}\right)=0$. Among such Lie algebras $\mathrm{g}(A)$ there exists a minimal due to the Kuratowski-Zorn lemma. Denote it by $\mathrm{g}\left(A_{\text {fin }}\right)$, where $A_{\text {fin }} \subset \Psi$.

If $\mathbf{F}=\mathbf{Q}\left(\left\{a_{j}: j \in \lambda\right\}\right), \lambda \subset \mathbf{N}$, then the field $\mathbf{F}$ is countable, since $\bigcup_{k=1}^{\infty} \aleph_{0}^{k}=\aleph_{0}$. If $B \subset \mathbf{K}$ and $\nu(B)=0$, where $\nu$ is a measure equivalent to the Haar measure on $\mathbf{K}$, then $\nu(B+\mathbf{F})=0$ and $\nu(B \mathbf{F})=0$ for a countable subfield $\mathbf{F}$ in $\mathbf{K}$, consequently, $\nu((B+\mathbf{F}) \mathbf{F})=0$ and $\nu\left(\bigcup_{k=1}^{\infty}\left(B^{k}+\mathbf{F}\right) \mathbf{F}\right)=0$, since $\nu\left(B_{1} B_{2}\right)=\int_{\mathbf{K}} \chi_{B_{1} B_{2}}(x) \nu(d x)$, where $\chi_{B}(x)=1$ for $x \in B$, $\chi_{B}(x)=0$ for $x \notin B, \chi_{B}$ denotes the characteristic function of a subset $B, B_{1}+B_{2}:=\{x$ : $\left.x=b_{1}+b_{2}, b_{1} \in B_{1}, b_{2} \in B_{2}\right\}, B_{1} B_{2}:=\left\{x: x=b_{1} b_{2}, b_{1} \in B_{1}, b_{2} \in B_{2}\right\}$.

Since $\nu^{n}\left(\mathrm{~g}\left(A_{\text {fin }}\right)\right)=0$, then $\nu\left(\mathbf{Q}\left(A_{\text {fin }}\right)\right)=0$, where $\nu_{\mathbf{K}}=\nu$ is the Haar measure on $\mathbf{K}$ as the additive group. Consequently, there exists $A_{\text {fin }}$ such that $\operatorname{card}(\Phi)=\mathrm{c}$, where $\Phi:=\Psi \backslash A_{\text {fin }}$, since each element $v$ from $\mathrm{g}$ is the finite linear combination over $\mathbf{Q}$ elements from $\gamma$, while in the Campbell-Hausdorff formula the expansion coefficients are rational (see $\S 11)$.

Over each field $\mathbf{F}=\left(\mathbf{Q}\left(A_{\text {fin }}\right)\right)(b)=\mathbf{Q}\left(A_{\text {fin }} \cup\{b\}\right)$ for $b \in \Phi$ consider a Lie subalgebra $\mathrm{g}\left(A_{\text {fin }}\right)_{\mathbf{F}}$ generated from the algebra $\mathrm{g}\left(A_{f i n}\right)$ by extension of the field of scalars $\mathbf{Q}\left(A_{f i n}\right)$ up to $\mathbf{F}, \mathrm{g}\left(A_{\text {fin }}\right)_{\mathbf{F}} \subset \mathrm{g}$, that is each element from $\mathrm{g}\left(A_{\text {fin }}\right)_{\mathbf{F}}$ is a finite linear combination over $\mathbf{F}$ elements from $\mathrm{g}\left(A_{\text {fin }}\right)$.

Each element $g \in U$ is a finite product of elements of local one-parameter subgroups of the form $\exp \left(t_{j} v_{j}\right), t_{j} \in \mathbf{K}$. The exponential mapping exp : $V \rightarrow U$ gives an uncountable family of local subgroups $S_{\mathbf{F}}=\exp \left(V \cap \mathrm{g}\left(A_{\text {fin }}\right)_{\mathbf{F}}\right)$, where $\mathbf{F}=\mathbf{Q}\left(A_{\text {fin }} \cup\{b\}\right), b \in \Phi$. All such local subgroups for different $\mathbf{F}=\mathbf{Q}\left(A_{\text {fin }} \cup\left\{b_{j}\right\}\right), b_{1} \neq b_{2} \in \Phi$, are pairwise isomorphic, since the fields $\mathbf{Q}\left(A_{\text {fin }} \cup\left\{b_{1}\right\}\right)$ and $\mathbf{Q}\left(A_{\text {fin }} \cup\left\{b_{2}\right\}\right)$ are pairwise isomorphic, and in $U$ there is satisfied the Campbell-Hausdorff formula about the local relation between the multiplication in the Lie algebra $g$ and the multiplication in its Lie group $G$ (see Chapters 2 and 3 in [4]). Moreover, $\mathrm{g}\left(A_{f i n}\right)_{\mathbf{Q}\left(A_{f i n} \cup\left\{b_{1}\right\}\right)}$ is isomorphic with $\mathrm{g}\left(A_{f i n}\right)_{\mathbf{Q}\left(A_{f i n} \cup\left\{b_{2}\right\}\right)}$. The isomorphism of the fields $\mathbf{Q}\left(A_{\text {fin }} \cup\left\{b_{1}\right\}\right)$ and $\mathbf{Q}\left(A_{\text {fin }} \cup\left\{b_{2}\right\}\right)$ is established by the mapping $\theta=\theta_{b_{1}, b_{2}}, \theta\left(b_{1}\right)=b_{2}$ with the identity mapping on the field $\mathbf{Q}\left(A_{\text {fin }}\right)$ such that $\theta\left(P_{k}\left(b_{1}\right) / L_{s}\left(b_{1}\right)\right)=P_{k}\left(b_{2}\right) / L_{s}\left(b_{2}\right)$, where $b_{1}, b_{2} \in \Phi, P_{k}$ and $L_{s}$ are non-zero polynomials of non-negative integer degrees $k$ and $s$ respectively with coefficients from the field $\mathbf{Q}\left(A_{\text {fin }}\right)$ (see also [1, 17]).

In the non-archimedean case each local subgroup $S_{\mathbf{F}}=: J_{\mathbf{F}}$ is the group, since $W=U$ is the group. In the case of the Lie group $G$ over $\mathbf{R}$ take $W=\bigcup_{n=1}^{\infty} U^{n}$ (see above). Therefore, $\bigcup_{n=1}^{\infty}\left(S_{\mathbf{F}}\right)^{n}=: J_{\mathbf{F}}$ is the subgroup in $G$. Then the group $J_{\mathbf{Q}\left(A_{f i n} \cup\{b\}\right)}$ is isomorphic with $J_{\mathbf{Q}\left(A_{f i n} \cup\{r\}\right)}$ for each $b \neq r \in \Phi$.

Take a bijective epimorphic mappings $\phi: \Phi \rightarrow \Phi$. It gives an isomorphism $\phi$ of each field $\mathbf{Q}\left(A_{\text {fin }} \cup\left\{b_{1}, \ldots, b_{z}\right\}\right)$ onto $\mathbf{Q}\left(A_{\text {fin }} \cup\left\{r_{1}, \ldots, r_{z}\right\}\right), \phi\left(P_{k}\left(b_{1}, \ldots, b_{z}\right) / L_{s}\left(b_{1}, \ldots, b_{z}\right)\right)=$ $P_{k}\left(r_{1}, \ldots, r_{z}\right) / L_{s}\left(r_{1}, \ldots, r_{z}\right)$ for each non-zero polynomials of integer non-negative degrees $k$ and $s$ of $z \in \mathbf{N}$ variables and with expansion coefficients from $\mathbf{Q}\left(A_{\text {fin }}\right)$, since $\phi(x)=x$ for each number $x$ from $\mathbf{Q}\left(A_{f i n}\right)$, where $r_{j}=\phi\left(b_{j}\right)$ for each $j=1, \ldots, z \in \mathbf{N}, b_{1}, \ldots, b_{z} \in \Phi$. Therefore, it has a natural extension up to an algebraic automorphism of the field $\mathbf{Q}(\Psi)=$ $\left(\mathbf{Q}\left(A_{\text {fin }}\right)\right)(\Phi)$ onto itself.

If $a \in \mathbf{K} \backslash \mathbf{Q}(\Psi)$, then the set $(\Psi, a)$ is algebraically dependent, that is there exist $b_{1}, \ldots, b_{k} \in \Psi$ such that the family $\left\{b_{1}, \ldots, b_{k}, a\right\}$ is algebraically dependent. This means that 
there exists a polynomial $F_{s}\left(T_{1}, \ldots, T_{k}, T_{k+1}\right)$ of a degree $s \in \mathbf{N}$ with rational expansion coefficients such that a substitution of $b_{1}, \ldots, b_{k}$ instead of $T_{1}, \ldots, T_{k}, a$ instead of $T_{k+1}$ the polynomial takes the zero value. Then for an automorphism $\phi$ of the field $\mathbf{K}$ a number $\phi(a)$ need to be a root of the polynomial $F_{s}\left(r_{1}, \ldots, r_{k}, T_{k+1}\right)$, where $r_{j}=\phi\left(b_{j}\right)$ for every $j=1, \ldots, k$. If $a$ is an algebraic number over $\mathbf{Q}$, then $k=0$ and there can be taken $\phi(a)=a$.

For each algebraic number $a$ from $\mathbf{K}$ over the field $\mathbf{Q}$ put $\phi(a)=a$. Since the field $\mathbf{Q}$ is everywhere dense in the field $\mathbf{R}$ or $\mathbf{Q}_{\mathbf{p}}$ respectively, while the fields $\mathbf{R}$ and $\mathbf{Q}_{\mathbf{p}}$ are complete as the normed spaces relative to their multiplicative norms, then $\mathbf{Q}$ is everywhere dense in $\mathbf{Q}(\Psi)$ relative to the norm inherited from the field $\mathbf{K}$. Therefore, every algebraic number from the field $\mathbf{K}$ over the field $\mathbf{Q}(\Psi)$ is the limit of a converging sequence of algebraic numbers from $\mathbf{K}$ over the field $\mathbf{Q}$. The field $\mathbf{C}$ is algebraically closed and is obtained by the way of the extension of the field $\mathbf{R}$ with the help of the root of the polynomial $x^{2}+1=0$, which is invariant relative to the automorphism $\phi$.

In the case of the non-archimedean local field $\mathbf{K}$ the residue class field $B(\mathbf{K}, 0,1) / B(\mathbf{K}, 0,|\pi|)$ is the finite field $\mathbf{F}_{q}$ with the number $q=p^{y}$ of its elements, where $y \in \mathbf{N}, \pi \in \mathbf{K}$, $|\pi|=\max \{|x|: x \in \mathbf{K},|x|<1\}, B\left(\mathbf{K}, x_{0}, r\right):=\left\{x \in \mathbf{K}:\left|x-x_{0}\right| \leq r\right\}$ is the ball of radius $r>0$ in $\mathbf{K}$, containing $x_{0}$. At the same time the residue class field $B\left(\mathbf{Q}_{\mathbf{p}}, 0,1\right) / B\left(\mathbf{Q}_{\mathbf{p}}, 0,1 / p\right)$ is composed of $p$ elements, where $|p|=1 / p$ for $p \in \mathbf{Q}_{\mathbf{p}}$. The field of $p$-adic numbers has the normalization group $\Gamma_{\mathbf{Q}_{\mathbf{p}}}:=\left\{|x|: x \neq 0, x \in \mathbf{Q}_{\mathbf{p}}\right\}=\left\{p^{k}: k \in \mathbf{Z}\right\}$. While $\Gamma_{\mathbf{K}}=\left\{p^{k / l}: k \in \mathbf{Z}\right\}$ for some $0<l \in \mathbf{N}$. That is $\mathbf{K}$ is obtained by a finite algebraic extension of the field of $p$-adic numbers by adding roots of polynomials with expansion coefficients from the field of rational numbers $\mathbf{Q}$ (see also Theorem 7 and Proposition 5 in §I.4 [26]).

Therefore, for each algebraic number $a \in \mathbf{K} \backslash \mathbf{Q}(\Psi)$ there exists a polynomial $F_{s}\left(b_{1}, \ldots, b_{v}, X\right)$ with $b_{1}, \ldots, b_{v} \in \Psi$ of degree $s \in \mathbf{N}$ with rational expansion coefficients such that $F_{s}\left(b_{1}, \ldots, b_{v}, a\right)=$ 0 , consequently, it can be taken $\phi(a)=c$, where $F_{s}\left(r_{1}, \ldots, r_{v}, c\right)=0, r_{j}=\phi\left(b_{j}\right), c \in \mathbf{K}$, since $\phi(q)=q$ for each rational number $q \in \mathbf{Q}$, while $\mathbf{Q}\left(b_{1}, \ldots, b_{v}\right)$ and $\mathbf{Q}\left(r_{1}, \ldots, r_{v}\right) \subset \mathbf{Q}_{p}$. Thus, the automorphism $\phi$ has an extension up to an automorphism $\phi: \mathbf{K} \rightarrow \mathbf{K}$ as in the case $\mathbf{K}=\mathbf{R}$, as well as for the local field $\mathbf{K}$.

Since $\operatorname{card}(\Phi)=\mathbf{c}, \operatorname{card} \mathbf{Q}(\Phi)=\mathbf{c}$ and $\mathbf{Q}(\Phi)$ is everywhere dense in $\mathbf{K}$, then there exist bijective surjective mappings $\phi: \Phi \rightarrow \Phi$ generating automorphisms of the field $\phi: \mathbf{K} \rightarrow \mathbf{K}$ as above such that for each open subsets $S$ and $T$ in $\mathbf{K}$ there is satisfied the relation for the cardinality $\operatorname{card}(\phi(S) \cap T)=\mathrm{c}$. Indeed, the set $\Phi$ can be described in the form of the disjoint union of subsets $\Lambda_{a}, a \in E, \operatorname{card}(E)=\mathrm{c}, \operatorname{card}\left(\Lambda_{a}\right)=\mathrm{c}$ for each $a, \bigcup_{a \in E} \Lambda_{a}=\Phi$, $\Lambda_{a} \cap \Lambda_{b}=\emptyset$ for each $a \neq b$. At the same time $\operatorname{card}\left(\mathbf{Q}\left(\Lambda_{a}\right) \cap T\right)=\mathrm{c}$ for each $T$ open in $\mathbf{K}$. Since $\operatorname{card}\left(\Lambda_{a}\right)=\operatorname{card}\left(\Lambda_{b}\right)$, then there exists a bijection $\phi_{b}^{a}: \Lambda_{a} \rightarrow \Lambda_{b}$ from $\Lambda_{a}$ onto $\Lambda_{b}$ for each $a, b$.

Take a bijective mapping $\eta: E \rightarrow E$ from $E$ onto $E$ such that $\eta(a) \neq b$ for each $a$. Then the combination of mappings $\left\{\phi_{\eta(a)}^{a}: a \in E\right\}$ generates the bijective mappings $\phi: \Phi \rightarrow \Phi$, putting $\phi(a)=a$ on $A_{\text {fin }}$, we get the bijective mapping $\phi: \Psi \rightarrow \Psi$ from $\Psi$ on $\Psi$. In view of the proof given above it has an extension up to an automorphism of the field $\phi: \mathbf{K} \rightarrow \mathbf{K}$. Therefore, $\operatorname{card}(\phi(S) \cap T)=\mathrm{c}$ for each $S$ and $T$ open in $\mathbf{K}$, since $\operatorname{card}\left(\mathbf{Q}\left(\Lambda_{a}\right) \cap T\right)=\mathrm{c}$ for each $T$ open in $\mathbf{K}$. It is not difficult to mention, that the family of such different algebraic automorphisms of the field $\mathbf{K}$ has the cardinality $2^{c}$, since $\operatorname{card}\left(\mathrm{c}^{c}\right)=2^{\mathrm{c}}[25]$. In view of Lemma 12 they are $\left(\mathcal{A}_{\nu}, \mathcal{B}(\mathbf{K})\right)$-non-measurable, where $\nu$ - is the non-negative non-trivial Haar measure on $\mathbf{K}$ (see also [3, 26]).

On the other hand, every automorphism $\phi$ of the field $\mathbf{K}$ generates an automorphism $f$ of the group $G_{0}:=\operatorname{gr}\left(J_{\mathbf{Q}\left(A_{f i n}\right)} \cup\left[\bigcup_{b \in \Phi} J_{\mathbf{Q}\left(A_{f i n} \cup\{b\}\right)}\right]\right)$, where $\operatorname{gr}(B)$ is a minimal algebraic subgroup in $G$ generated by finite products $g_{1}^{a_{1}} \ldots g_{r}^{a_{r}}$ of all elements $g_{j} \in B, a_{j} \in \mathbf{Z}, j=1, \ldots, r \in$ 
$\mathbf{N}$. The automorphism $f$ is produced with the help of the isomorphisms $f: J_{\mathbf{Q}\left(A_{f i n} \cup\{b\}\right)} \rightarrow$ $J_{\mathbf{Q}\left(A_{f i n} \cup\{\phi(b)\}\right)}$ for each $b \in \Phi$, while the restriction $f \mid J_{\mathbf{Q}\left(A_{f i n}\right)}$ take, for example, the identity mapping. The mapping $f$ has an extension from $A:=J_{\mathbf{Q}\left(A_{f i n}\right)} \cup\left[\bigcup_{b \in \Phi} J_{\mathbf{Q}\left(A_{f i n} \cup\{b\}\right)}\right]$ on $G_{0}$ by taking of all possible finite products of initial elements from $A$. For each such subgroup $G_{0}$ of the group $G$ the automorphism has an extension on $G$, since every automorphism $q$ of a subgroup $Y$ of the group $G$ can be extended up to an automorphism of some group $H$ containing in itself $G$, but $q(G)$ is contained in $H$ and is isomorphic with $G$ (see [19, 16]).

By the construction of the group $G_{0}$ it is everywhere dense in the group $W$ in the topology inherited from $G$. Then due to Lemma 12 the automorphism $f$ is $\left(\mathcal{A}_{\mu}, \mathcal{B}(G)\right)$ non-measurable. This also follows from the fact that the exponential mapping exp from the neighborhood of zero $V_{0}$ in the Lie algebra $g$ on the neighborhood $U_{e}$ of the unit element in $G$ induces the image of the measure $\nu_{\exp }^{n}$ on $U_{e}$, where $n$ is the dimension of $\mathrm{g}$ as the linear space over the field $\mathbf{K}, \nu^{n}$ is the Haar measure on $\mathbf{K}^{\mathbf{n}}$ as the additive group. At the same time the measure $\nu_{\exp }^{n}$ is equvalent to the restriction of the Haar measure $\mu$ on $U_{e}$ (see Definitions 1 above).

From the proof of Theorem 13 it follows the following.

14. Corollary. The family $\Upsilon$ of non-measurable automorphisms from Theorem 13 has a subfamily $\Omega$ of the cardinality card $(\Omega) \geq 2^{\mathrm{c}}$ such that every $f \in \Omega$ after a restriction on each one-parameter subgroup over the field $\mathbf{K}$ in $G$ is non-measurable relative to the corresponding Haar measure on the subgroup.

15. Theorem. Let $\mathrm{g}$ be a non-trivial Lie algebra finite-dimensional over the field $\mathbf{K}$ with a measure $\mu$ equal to the non-trivial non-negative Haar measure on the additive group for $\mathrm{g}$. Then the algebra $\mathrm{g}$ has $2^{\mathrm{c}}$ non-measurable automorphisms.

Proof. Take any algebraic automorphism $\phi$ of the field $\mathbf{K}$ from the proof of Theorem 13. Since $\mathbf{g}$ is finite-dimensional over the field $\mathbf{K}$, then the non-negative non-trivial Haar measure $\mu$ on $\mathrm{g}$ as the additive group for $\mathrm{g}$ is equivalent to the measure $\nu^{n}$ (see Definitions 1 ). The automorphism $\phi$ has the extension up to an automorphism of the algebra: $\phi\left(a_{j} v_{j}\right)=\phi\left(a_{j}\right) v_{j}$, $\phi\left(a_{1} v_{1}+\ldots+a_{m} v_{m}\right)=\phi\left(a_{1}\right) v_{1}+\ldots+\phi\left(a_{m}\right) v_{m}, \phi\left(\left[a_{k} v_{k}, a_{j} v_{j}\right]\right)=\left[\phi\left(a_{k}\right) v_{k}, \phi\left(a_{j}\right) v_{j}\right]$ for each $a_{j} \in \mathbf{K}, k, j=1, \ldots, m$, where $v_{1}, \ldots, v_{m}$ is the basis of generators in $\mathrm{g}$ (see Definitions 2.1). Therefore, in view of Lemma 12 the automorphism $\phi$ is $\left(\mathcal{A}_{\mu}, \mathcal{B}(\mathrm{g})\right)$-non-measurable, relative to the additive group of the algebra $g$. The family of such different automorphisms of the algebra $g$ has the cardinality $2^{\mathrm{c}}$.

16. Theorem. Let $G$ be a locally compact Hausdorff group with a countable base of neighborhoods of the unit element $e$ and $f: G \rightarrow G$ be its automorphism non-measurable relative to the non-trivial non-negative left- (or right-)invariant Haar measure $\mu$ on $G$. Then $G$ has a topologically irreducible unitary representation, which is not weakly measurable.

Proof. Since $G$ has a non-measurable automorphism, then it is non-discrete, since all algebraic automorphisms are continuous relative to the discrete topology, since in discrete topology every point is an open subset. On the other hand, every $T_{0}$ topological group is completely regular (see Theorem 8.4 [23]). In view of Theorem 5.8 [23] a subgroup of a topological group is discrete if and only if it contains an isolated point. Therefore, the group $G$ is dense in itself, that is every its point $q$ is a limit of a convergent net, contained in the punctured open neighborhood $U \backslash\{q\}$ of a point $q$. In view of Lemma 5.28 [23] locally countably compact regular topological space $Y$ can not be presented as a countable union of closed subsets with the empty interior. Thus, every open subset $U$ in $G$ is uncountable, $\operatorname{card}(U) \geq \mathrm{c}$ (see Remark (4.26) [23]). In view of this the Haar measure $\mu$ on $G$ has not atoms. By its construction the Haar measure is Borel regular.

Recall, that the unitary representation is a homomorphism $T: G \rightarrow U(X)$, where $U(X)$ is the unitary group of the Hilbert or the unitary space $X$ over the field of complex numbers C. It is called topologically irreducible, if in $X$ there does not exist any closed invariant 
subspace relative to the family of unitary operators $\left\{T_{g}: g \in G\right\}$, besides $\{0\}$ and the entire $X$.

A unitary representation is called weakly measurable, if the functions $\left(y, T_{g} z\right)$ are $\left(\mathcal{A}_{\mu}, \mathcal{B}(\mathbf{C})\right)$ measurable for each given vectors $y, z \in X$, where $g \in G$. On every locally compact group there exists a non-trivial non-negative left-(or right-)invariant Haar measure (see $§ 27$ [14] and $[3,23]$ ).

Then the space $L^{1}(G, \mathcal{A}, \mu, \mathbf{C})$ is supplied with the ring structure with the usual addition of functions and convolutions of functions as multiplication in the ring, which is called the group ring (see $\S 28$ [14]). A ring $R$ is called normed, if it is the normed space, for each $x, z \in R:|x z| \leq|x||z|$, and if in $R$ there is the unit $e$, then $|e|=1$. The complete normed ring is called the Banach ring. A ring $R$ is called symmetric, if it is supplied with the involution $x \mapsto x^{*}$, which maps from $R$ into $R$. In particular, the $\operatorname{ring} L^{1}(G, \mathcal{A}, \mu, \mathbf{C})$ is symmetric.

Therefore, a representation of the group generates a representation of the group ring in the ring (algebra in more modern terminology) $L(X)$ of bounded linear operators from $X$ into $X$. The adjoining of the unit to the $\operatorname{ring} L^{1}(G, \mathcal{A}, \mu, \mathbf{C})$ gives the group ring with the unit, denote it by $R(G)$. In view of Theorem 29.1 [14] to each representation $x \mapsto A_{x}$ of the group ring $R(G)$ not containing a degenerate representation, there corresponds a continuous unitary representation $g \mapsto T_{g}$ of the group $G$. Vice versa, to every weakly continuous unitary representation $g \mapsto T_{g}$ of the group $G$ there corresponds a representation $x \mapsto A_{x}$ of its group ring $R(G)$, which does not contain a degenerate representation. These representations are related with each other by the formula: $A_{b e+f}=b 1+\int_{G} f(g) T_{g} \mu(d g)$ for each $b \in \mathbf{C}, f \in L^{1}(G, \mathcal{A}, \mu, \mathbf{C})$.

A linear functional $f$ is called positive, if $f\left(x^{*} x\right) \geq 0$ for each $x \in R$. If $f_{1}$ and $f$ are positive functionals, then $f_{1}$ is called subordinated to the functional $f$, which is denoted by $f_{1}<f$, if there exists a number $b$ such that $b f-f_{1}$ is a positive functional in $R$. A functional $f_{1}$ in a symmetric ring is called subordinated to a given positive functional $f$, if $f_{1}$ is a linear combination with complex coefficients of positive functionals subordinated to the functional $f$. A positive functional $f$ is called indecomposable, if each functional $f_{1}$ subordinated to the functional $f$ is its multiple, that is $f_{1}=b f$, where $b \in \mathbf{C}$. A representation $x \mapsto A_{x}$ is called cyclic, if there exists a vector $y_{0} \in X$ such that $\left\{A_{x} y_{0}: x \in R\right\}$ is everywhere dense in $X$.

In view of Theorem 19.3.1 [14] a cyclic representation of a Banach symmetric ring $R$ : $x \mapsto A_{x}$ is irreducible if and only if, each defining it positive functional $f(x)=\left(A_{x} y_{0}, y_{0}\right)$ is indecomposable. Let $S$ be a set of all positive functionals on $R$ such that $f(e)=1$. In view of Proposition 19.4.1 [14] a positive functional $f$, satisfying condition $f(e)=1$ is indecomposable if and only if it is an extremal point in the set $S$.

If $H$ is a subgroup in $G$ and $M$ is a topologically irreducible unitary representation of $H$ in the Hilbert space $Y, Y \ni y_{0} \neq 0,\left\|y_{0}\right\|=1, t(h):=\left(M_{h} y_{0}, y_{0}\right), h \in H$, then the function $t$ is positive definite. Consider a convex subset $W$ of all positive definite functions on $G$, coinciding with $t$ on $H$. This set is non-void, since it contains the function equal to $t$ on $H$ and 0 on $G \backslash H$. If $v \in W$, then $v(e)=\left(T_{e} y_{0}, y_{0}\right)=1$, consequently, $|v(g)| \leq 1$ for every $g \in G$. Therefore, $W$ is compact in the topology of pointwise convergence. In view of the Krein-Milman theorem $W$ contains an extremal point $r$ (see Theorem 3.9.1 [14]), its restriction is $\left.r\right|_{H}=f$. Consequently, $r$ is indecomposable and to it a topologically irreducible unitary representation of the group $G$ corresponds (see $\S 2.1$ [2], 14, 21, 23]).

Consider the Hilbert space $X:=L^{2}(G, \mathcal{A}, \mu, \mathbf{C})$ of the equivalence classes of all functions $v: G \rightarrow \mathbf{C}$ with square integrable its module on $G$ relative to the measure $\mu$. Then there exists a strongly continuous unitary regular representation $T: G \rightarrow U(X)$. The strong 
continuity means, that $T_{g} z$ is the continuous mapping from $G$ into $X$ for each $z \in X$, where $X$ is supplied wit the standard norm associated with the scalar product: $\|z\|^{2}=(z, z)$. In the case of the left-invariant Haar measure it is given by the formula $T_{g} v(h):=v\left(g^{-1} h\right)$ for each $g, h \in G, v \in X$, where $\mu(g J)=\mu(J)$ for every $g \in G$ and each $\mu$-measurable subset of finite measure, $J \in \mathcal{A}$.

Evidently, if a mapping (in the given case a functional $\left(T_{g} x, y\right)$ of the representation $T$ ) is non-measurable on an open subset $W$ in $G$, then it is non-measurable on $G$. For the proof of non-measurability it is sufficient to take a clopen subgroup $W$ in $G$, which is compact, if $G$ is totally disconnected, or $W=\bigcup_{n=1}^{\infty} U^{n}$ for a locally connected $G$ which is not totally disconnected, where $U$ is a symmetric open neighborhood of $e$ in $G, 0<\mu(U)<\infty$ (see Theorems 7.7 and 5.7 [23]). Since $e$ has the countable base of neighborhoods of $e$, then the spaces $L^{p}(W, \mathcal{A}, \mu, \mathbf{C})$ for $1 \leq p<\infty$ are separable. Then the proof reduces to the consideration of the subgroup $W$. Denote $W$ by $G$.

The regular representation $T$ is injective, $T_{g} \neq T_{h}$ for each $g \neq h \in G$. In view of Theorem 41.4.3 [14] it can be decomposed into the direct integral of topologically irreducible unitary representations $T=\bigoplus \int_{S} T^{s} \lambda(d s)$, where $S$ is a compact (bi-compact in old terminology) Hausdorff topological space, where $\lambda$ is a $\sigma$-additive measure on $\mathcal{B}(S)$ (see also [25, 14, 23]). At the same time the representation $T^{s}$ is strongly continuous. Therefore, $q^{-1}(\mathcal{B}(G)) \subset \mathcal{B}(X)$ for every function $q(g):=T_{g}^{s} z$ for marked $z \in X, s \in S$, where $q: G \rightarrow X$.

For each non-zero vector $z \in X^{s}, z \neq 0$, the closure of the linear span over the field of complex numbers $\mathbf{C}$ of all vectors $T_{g}^{s} z$ coincides with $X^{s}$, where $X^{s}$ is an invariant closed subspace in $X$ relative to the unitary representation $T^{s}$. Then the set of functions $\left\{q_{x, z, s}\right.$ : $\left.G \rightarrow \mathbf{C} ; x \in X^{s}\right\}$ separates points in $X^{s}$, where $q_{x, z, s}(g):=\left(x, T_{g}^{s} z\right), x, z \in X^{s}$. At the same time $q_{x, z, s} \circ f(g)=\left(x, T_{f(g)}^{s} z\right)$. If $A \subset \mathbf{C}$, then $\left(q_{x, z, s} \circ f\right)^{-1}(A)=f^{-1}\left(q_{x, z, s}^{-1}(A)\right)$. If $A$ is open in $\mathbf{C}$, then $q_{x, z, s}^{-1}(A)$ is open in $G$. Since $f$ is non-measurable on $G$, also $G \ni h \mapsto g h \in G$ is the continuous mapping from $G$ onto $G, g U$ is open for each $g \in G$ and open $U$ in $G$, then the restriction $\left.f\right|_{U}$ is non-measurable for each $U$ open in $G$.

Consider an algebraic homomorphism $T \circ f: G \rightarrow U(X)$. If every $T^{s} \circ f$ would be weakly $\left(\mathcal{A}_{\mu}, \mathcal{B}(\mathbf{C})\right)$-measurable, then $T \circ f$ also would be weakly $\left(\mathcal{A}_{\mu}, \mathcal{B}(\mathbf{C})\right)$-measurable. But in view of strong continuity of $T$ and non-measurability of the automorphism $f$ the composition $T \circ f$ is not weakly $\left(\mathcal{A}_{\mu}, \mathcal{B}(\mathbf{C})\right.$ )-measurable (see also Lemma 12 and Theorem 13 above).

17. Corollary. If $G$ is a non-trivial locally compact Lie group, then it has not less than $2^{c}$ weakly non-measurable relative to a non-trivial non-negative Haar measure $\mu$ topologically irreducible unitary representations.

18. Remark. From the proofs of Theorems 13 and 16 it follows, that the property of $\mu$-non-measurability of an automorphism $f$ or of a unitary representation is local: if $f$ is $\mu$-non-measurable for the restriction on an open subset $W$ in $G$, then it is $\mu$-non-measurable on $G$.

For a totally disconnected locally compact group it can be taken as $W$ a clopen compact subgroup in $G$ (see Theorem $7.7[23]$ ). In the case of locally connected which is not totally disconnected group $G$ it can be taken a clopen subgroup $W=\bigcup_{n=1}^{\infty} U^{n}$, where $0<\mu(U)<$ $\infty, U$ is a connected symmetric open neighborhood of $e$ in $G$ (see Theorem 5.7 [23]). Then on $W$ there exists a probability measure equivalent with $\mu$ (see also Lemmas 2 and 3). In the case of a locally compact Lie group over $\mathbf{R}$ it can be taken on $U$ also a measure equivalent with the measure $\lambda_{\theta^{-1}}$, where $\theta: U \rightarrow\left(S^{1}\right)^{n}$ is the topological embedding from Lemma 11, while $\lambda$ is the Haar measure on $\left(S^{1}\right)^{n}, \lambda_{\theta^{-1}}(Y):=\lambda(\theta(Y))$ for each $Y \in \mathcal{B}(U)$.

In Theorem 16 and Lemma 17 there are irreducible unitary representations, since in general one can not restrict on characters because of Lemmas 5, 8 and Corollaries 6,9. 
19. Let $G$ be a $C^{\infty}$ or $C^{\omega}$ non-trivial Lie group over the field $\mathbf{K}=\mathbf{R}$ or a non-archimedean local field $\mathbf{K}$, moreover, $G$ is complete as an uniform space. Suppose that $\mu$ is a $\sigma$-additive $\sigma$-finite non-negative non-trivial Borel regular measure on $\mathcal{B}(G)$ such that for each $g \in G$ there exists an open neighborhood $U, g \in U$, with $0<\mu(U)<\infty$, moreover, $\mu$ has not any atom. Let exp : $V \rightarrow U$ be the exponential mapping for $G$ as the smooth manifold over $\mathbf{K}$ from the open neighborhood $V$ of zero in $T_{e} G$ onto an open neighborhood $U$ of the unit element $e$ in $G$. Let also a measure $\nu$ on $T_{e} G$ be such that $\nu(J)=\mu(\exp (J))$ for each Borel subset $J \in \mathcal{B}\left(T_{e} G\right)$, where $T_{e} G$ is the linear space of separable type over the field $\mathbf{K}$.

Suppose that $G$ has an open neighborhood $U$ of the unit element $e$ with $0<\mu(U)<\infty$, $\exp : V \rightarrow U$ for which a set of elements $S:=\{g \in U: g$ belongs to a local one-parameter subgroup in $U$, over the field $\mathbf{K}$, moreover it is unique for a given $g$ \} has a positive outer measure, $\mu^{*}(S)>0$, where local one-parameter subgroups have the form $\{\exp (x v): x \in$ $\mathbf{K},|x|<\epsilon\} \subset U, v \in T_{e} G, \epsilon>0$. Suppose that the restriction of $\ln$ on $U$ corresponds to the Campbell-Hausdorff formula, where $\ln$ is the inverse mapping to exp.

Let $\pi_{v}: T_{e} G \rightarrow \mathbf{K} v$ be a linear over $\mathbf{K}$ projection operator, moreover, $\nu_{v}$ is equivalent to the Haar measure on $\mathbf{K}$, where $v$ is a non-zero vector of the tangent space $v \in T_{e} G, \mathbf{K} v$ is the one-dimensional over $\mathbf{K}$ subspace in $T_{e} G$ containing a vector $v, \nu_{v}(J)=\nu\left(\pi_{v}^{-1}(J)\right)$ for each $J \in \mathcal{B}(\mathbf{K})$.

Theorem. Then such group $G$ has a family $\Upsilon$ of $\left(\mathcal{A}_{\mu}, \mathcal{B}(G)\right)$-non-measurable automorphisms of the cardinality not less than $2^{\mathrm{c}}, \operatorname{card}(\Upsilon) \geq 2^{\mathrm{c}}$. Moreover, $\Upsilon$ has a subfamily $\mathcal{P}$ of automorphisms $f$, restrictions of which on one-parameter over $\mathbf{K}$ local subgroups $\{\exp (x v):|x|<\epsilon\}$ in $S$ are non-measurable relative to the corresponding Haar measure on $\{\exp (x v):|x|<\epsilon\}$.

Proof is proceeded by the generalization of the proof of Theorem 13. For this consider a minimal subgroup $G_{S}$ in $G$, generated by elements in $S$. The image $\nu$ on $V$ of the measure $\mu$ with the help of the logarithmic mapping $\ln , \nu(B)=\mu(\exp (B))$ for every $B \in \mathcal{B}(V)$, has the extension up to a $\sigma$-additive finite measure on $\mathrm{g}, \nu(B):=\sum_{j=1}^{\infty} \nu\left(\left(B-h_{j}\right) \cap V\right) / 2^{j}$ for every $B \in \mathcal{B}(\mathrm{g})$, where $\left\{\left(V+h_{j}\right): j \in \mathbf{N}, h_{j} \in \mathrm{g}\right\}$ is the covering of g. Therefore, $\nu$ has $\sigma$ additive projections $\nu_{\omega\left(\mathbf{K}^{\mathbf{n}}\right)}$ on $\omega\left(\mathbf{K}^{\mathbf{n}}\right)$ for each embedding $\omega: \mathbf{K}^{\mathbf{n}} \hookrightarrow \mathbf{g}$ as the $\mathbf{K}$-linear space, $\nu_{\omega\left(\mathbf{K}^{\mathbf{n}}\right)}(B)=\nu\left(\pi^{-1}(B)\right)$ for each $B \in \mathcal{B}\left(\omega\left(\mathbf{K}^{\mathbf{n}}\right)\right)$, where $\pi: \mathrm{g} \rightarrow \omega\left(\mathbf{K}^{\mathbf{n}}\right)$ is the projection.

The operator $\pi$ is $\mathbf{K}$-linear and it exists, since $\omega\left(\mathbf{K}^{\mathbf{n}}\right)$ is finite-dimensional over $\mathbf{K}$, the field $\mathbf{K}$ is locally compact (see Theorems 5.13 and 5.16 [20] and [15]). The image $\left.\nu_{\omega(\mathbf{K})}\right|_{V \cap \omega(\mathbf{K})}$ with the help of exp generates the measure on a local one-parameter subgroup in $S, \mu_{g}(B)=$ $\nu_{\omega(\mathbf{K})}(\ln (B))$ for every $B \in \mathcal{B}\left(g_{W} \cap U\right)$, which has the extension up to the $\sigma$-additive measure $\mu_{g}$ on $g_{W}$. Therefore, the set of elements of finite orders from $g_{W}$ has $\mu_{g}$-measure zero. Then as in $\S 13 \mu^{*}\left(\left(G_{S}\right)_{f i n} \cap S\right)=0$, since the measure $\mu$ is Borel regular, $\sigma$-additive and it has not atoms. Since the measure $\mu$ is $\sigma$-finite, then also $\mu^{*}\left(\left(G_{S}\right)_{f i n}\right)=0$.

Consider the Lie algebra $\mathbf{g}$ generated by $\ln (S)$ over the field $\mathbf{K}$. As the linear space $\mathbf{g}$ has the separable type over $\mathbf{K}$, that is there exists a countable family $\rho$ of $\mathbf{K}$-linearly independent vectors the linear span of which $\operatorname{span}_{\mathbf{K}} \rho$ is everywhere dense in $\mathbf{g}$. Then the minimal Lie algebra over $\mathbf{K}$ Liealg $\left(\operatorname{span}_{\mathbf{K}} \rho\right)$ containing $\operatorname{span}_{\mathbf{K}} \rho$ is everywhere dense in $\mathbf{g}$.

Construct $\mathrm{g}_{\text {fin }}$ as the minimal algebra over the field of rational numbers $\mathbf{Q}$ generated by $\ln \left(\left(G_{S}\right)_{f i n} \cap S\right)=0$ and $\rho$. For every minimal subalgebra $\mathrm{g}\left(\left\{v_{j}: j \in \lambda\right\}\right)$ over $\mathbf{K}$ with generators $v_{j} \in \rho, \lambda \subset \mathbf{N}$, there exists $A \subset \Psi$ such that $\mathrm{g}\left(\left\{v_{j}: j \in \mathbf{N}\right\}\right) \cap \mathrm{g}_{\text {fin }} \subset \mathrm{g}(A)$, where $\mathrm{g}(A)$ is the minimal Lie algebra over the field $\mathbf{Q}(A)$, satisfying this inclusion, $\gamma_{A}$ denotes the Hamel basis $\mathrm{g}(A)$ over $\mathbf{Q}(A)$, since every $v$ from $\mathrm{g}$ is the finite linear combination over $\mathbf{Q}$ of elements from $\gamma$, also $\operatorname{card}\left(\bigcup_{k=1}^{\infty}\left(\gamma \aleph_{0}\right)^{k}\right)=\operatorname{card}\left(\gamma \aleph_{0}\right)=\operatorname{card}(\gamma) \geq \mathrm{c}$, while expansion coefficients in the Campbell-Hausdorff formula are rational numbers, $\gamma$ denotes the Hamel basis of $\mathrm{g}$ over $\mathbf{Q}$ as the $\mathbf{Q}$-linear space, $\operatorname{card}(\Psi \backslash A)=\mathrm{c}$. 
If $B \subset \mathbf{K}$ and $\nu(B)=0$, where $\nu$ is a measure equivalent with the Haar measure on $\mathbf{K}$, then $\nu(B+\mathbf{F})=0$ and $\nu(B \mathbf{F})=0$ for a countable subfield $\mathbf{F}$ in $\mathbf{K}$, consequently, $\nu((B+\mathbf{F}) \mathbf{F})=0$ and $\nu\left(\bigcup_{k=1}^{\infty}\left(B^{k}+\mathbf{F}\right) \mathbf{F}\right)=0$, since $\nu\left(B_{1} B_{2}\right)=\int_{\mathbf{K}} \chi_{B_{1} B_{2}}(x) \nu(d x)$, where $\chi_{B}(x)=1$ for $x \in B, \chi_{B}(x)=0$ for $x \notin B, \chi_{B}$ is the characteristic function of a subset $B$, $B_{1}+B_{2}:=\left\{x: x=b_{1}+b_{2}, b_{1} \in B_{1}, b_{2} \in B_{2}\right\}, B_{1} B_{2}:=\left\{x: x=b_{1} b_{2}, b_{1} \in B_{1}, b_{2} \in B_{2}\right\}$.

Since $\mu^{*}\left(\left(G_{S}\right)_{\text {fin }}\right)=0$ and $\nu_{\mathrm{g}}^{*}\left(\mathrm{~g}_{\text {fin }}\right)=0, \operatorname{card}(\rho) \leq \aleph_{0}$, then there exists $A_{\text {fin }} \subset \Psi$ such that $\mathrm{g}\left(A_{\text {fin }}\right) \supset \mathrm{g}_{\text {fin }}$ and $\operatorname{card}\left(\Psi \backslash A_{\text {fin }}\right)=\mathrm{c}$, since $\beta \aleph_{0}=\beta$ for every cardinal number $\beta \geq \aleph_{0}$, moreover, $\nu_{\omega(\mathbf{K})}\left(\mathbf{Q}\left(A_{f i n}\right)\right)=0$, since $\nu_{\omega(\mathbf{K})}(B)>0$ for each $B$ open in $\omega(\mathbf{K}), \nu_{\omega(\mathbf{K})}$ is equivalent to the Haar measure on $\mathbf{K}$ due to the conditions from $\S 19$.

Take $\Phi=\Psi \backslash A_{\text {fin }}$ and an algebraic automorphism $\phi$ of the field $\mathbf{K}$ from $\S 13$. Analogously to $\S 13$ we construct an automorphism $f$ of the group $G_{S}$, also it has an extension up to an automorphism of the group $G$ (see [16, 19]). In view of Lemma 12 and $\mu^{*}(S)>0$ it follows, that $f$ is $\left(\mathcal{A}_{\mu}, \mathcal{B}(G)\right)$-non-measurable. Non-measurability of restrictions of $f$ on oneparameter subgroups relative to the Haar measure on them follows from the properties of $\phi$ as in $\S 13$.

20. Theorem. Let $G$ be an infinite topological dense in itself Hausdorff group with a non-negative non-trivial Borel regular measure $\mu$ on $\mathcal{B}(G)$ having not any atom, also for each $g \in G$ there exists an open neighborhood $U$ such that $0<\mu(U)<\infty$, moreover, $G$ is complete as the uniform space, card $(U) \geq \mathrm{c}$ for each open $U$ in $G$. Then there exists a family $\Upsilon$ of $\left(\mathcal{A}_{\mu}, \mathcal{B}(G)\right)$-non-measurable different automorphisms of the group $G$ of the cardinality $\operatorname{card}(\Upsilon) \geq 2^{\mathrm{c}}$.

Proof. Take an open symmetric neighborhood $U$ of the unit element $e$ in $G$ such that $0<\mu(U)<\infty$, then $\mu$ is the $\sigma$-finite measure on $W=\bigcup_{n=1}^{\infty} U^{n}$ (see Theorem $7.4[23]$ ). In view of the fact that $G$ is infinite and dense in itself, then it is non-discrete (see Theorem 5.8 [23]). If $\left\{g_{n}: n \in \alpha\right\}$ is a net converging to $e, \alpha$ is an ordinal, $\operatorname{card}(\alpha) \geq \aleph_{0}$, then $\left\{g g_{n}: n \in \alpha\right\}$ is the net converging to $g$ for each $g \in G$.

The topological Hausdorff group $G$ can be supplied with the left-invariant uniformity giving the initial topology on $G$, while the left-invariant uniformity can be produced with the help of the family $\left\{\eta_{x}: x \in M\right\}$ left-invariant pseudo-metrics $\eta_{x}(g, h)=\eta_{x}\left(h^{-1} g, e\right)$ for each $g, h \in G$, then $\eta_{x}\left(g^{a}, g^{b}\right)=\eta_{x}\left(g^{a-b}, e\right)$ for each $g \in G$ and each $a, b \in \mathbf{Z}$, where $M$ is some set (see Chapter 8 in [25]).

If $g \in G, \operatorname{ord}(g)=k<\infty$, then $\operatorname{ord}\left(g^{r}\right) \leq k$ for each $r \in \mathbf{Z}$, in particular, for mutually prime numbers $r$ and $k$ there is satisfied $\operatorname{ord}(g)=\operatorname{ord}\left(g^{r}\right)$. If $\operatorname{ord}(g)=\omega_{0}$, then $\operatorname{ord}\left(g^{r}\right)=\omega_{0}$ for each integer non-zero $r$, where $\omega_{0}$ denotes the initial ordinal of the cardinality $\aleph_{0}$.

Since the measure $\mu$ is non-trivial, non-negative and for $e \in G$ there exists an open neighborhood such that $0<\mu(U)<\infty$, the set $\mathbf{N} \cup\left\{\omega_{0}\right\}$ is countable, then there exists $k \in \mathbf{N} \cup \omega_{0}$ such that the outer measure of the intersection is positive $\mu^{*}\left(U \cap G_{k}\right)>0$, where $G_{k}=\operatorname{gr}\{g \in G: \operatorname{ord}(g)=k\}$ is the minimal subgroup in $G$ generated by elements of the $k$-th order. Therefore, it is sufficient to consider all such $G_{k}$ in the topology inherited from $G, \mu^{*}\left(U \cap G_{k}\right)>0$.

Mention that if $Y$ is an everywhere dense in $G$ subgroup, also $\operatorname{card}(B \cap T) \geq \mathrm{c}$ for some subset $B$ in $G$ for each $T$ from the base $\Pi$ of neighborhoods of the unit element $e \in G$, $T \in \Pi$, then $\operatorname{card}((B Y) \cap P) \geq \mathrm{c}$ for each $P$ open in $G$.

Consider the family $\Xi$ consisting of subgroups $H$ in $G$ and their automorphisms $s: H \rightarrow$ $H$ such that $\operatorname{card}(H) \geq \mathrm{c}, \operatorname{card}(s(P) \cap T) \geq \mathrm{c}$ for each $P$ and $T$ open in $H$ in the topology inherited from $G$. Such $H$ exists. For the proof of their existence take $U$ and $W$ as above. Let $W_{k}:=\{g \in W: \operatorname{ord}(g)=k\}$, where $k \in \mathbf{N} \cup \omega_{0}$. Then for at least one $k$ there is satisfied the equality $\operatorname{card}\left(W_{k}\right)=\operatorname{card}(W)$, since $\operatorname{card}(W) \geq \mathrm{c}$.

Since $\operatorname{card}(U) \geq \mathrm{c}$ and every subgroup $\left\{g^{n}: n \in \mathbf{Z}\right\}$ generated by a chosen element 
$g \in G$ is either finite or countable, then in $W$ there exists a family $J_{b, k}, b \in \Lambda, k \in \mathbf{N} \cup \omega_{0}$, $\operatorname{card}(\Lambda) \geq \mathrm{c}, \operatorname{card}\left(J_{b}\right)=\operatorname{card}(U) \geq \mathrm{c}$. This family can be chosen such that elements in $J_{b, k}$ would be algebraically independent: $g \in J_{b, k}$ can not be presented as a finite product of elements different from it from the set $J_{b, k}$, moreover, $\operatorname{gr}\left(J_{b, k}\right) \cap \operatorname{gr}\left(J_{d, l}\right)=\{e\}$ for each $b \neq d$, since $\mathbf{q}^{\aleph_{0}}=\mathbf{q}$ for each $\mathbf{q} \geq \mathbf{c}$, where $J_{b}=\bigcup_{k \in \mathbf{N} \cup \omega_{0}} J_{b, k}, \mathbf{q}$ and $\mathbf{c}:=\operatorname{card}(\mathbf{R})$ are cardinal numbers, $\operatorname{gr}(B)$ denotes the minimal subgroup in $G$ generated by elements from $B$, every $g$ from $J_{b, k}$ has the order $\operatorname{ord}(g)=k$. Moreover, $J_{b, k}$ can be chosen such that $G \backslash G_{\Lambda} \supset(Y \backslash\{e\})$, where $Y$ is some everywhere dense in $G$ subgroup $G_{\Lambda}:=\operatorname{gr}\left(\bigcup_{b \in \Lambda, k \in \mathbf{N} \cup \omega_{0}} J_{b, k}\right)$, since $G_{\Lambda}$ and $Y$ have algebraically indeppendent from each other families of generating elements, $G_{\Lambda} \cap Y=\{e\}$.

Choose every $J_{b}$ such that $\operatorname{card}\left(J_{b, k_{0}} \cap P\right) \geq$ c for each $P$ open in $W$ for at least one $k_{0} \in \mathbf{N} \cup \omega_{0}$. Make it for every $k_{0} \in \mathbf{N} \cup \omega_{0}$, for which $\operatorname{card}\left(\left\{g \in W: \operatorname{ord}(g)=k_{0}\right\}\right) \geq \mathbf{c}$. Let $\phi: \Lambda \rightarrow \Lambda$ be a bijective mapping from $\Lambda$ onto $\Lambda, \phi(\Lambda(k))=\Lambda(k), \Lambda=\bigcup_{k \in \mathbf{N} \cup \omega_{0}} \Lambda(k)$. Let $S_{b, k_{0}} \subset J_{b, k_{0}}$ and $\operatorname{card}\left(S_{b, k_{0}}\right)=\operatorname{card}\left(S_{d, k_{0}}\right) \geq \mathrm{c}$ for each $b, d \in \Lambda\left(k_{0}\right) \subset \Lambda$. With the help of the transfinite induction take $S_{b, k_{0}}$ and bijective mappings $\psi_{b, k_{0}}^{d}: S_{d, k_{0}} \rightarrow S_{b, k_{0}}$ from $S_{d, k_{0}}$ onto $S_{b, k_{0}}$, put $s(g):=\psi_{\phi(d), k_{0}}^{d}(g)$ for each $g \in S_{d, k_{0}}$ and with the help of finite products of elements in $G$, take $\left.s\right|_{Y}=i d$, extend $s$ from $Y \cup \bigcup_{b \in \Lambda\left(k_{0}\right), k_{0} \in \mathbf{N} \cup \omega_{0}} S_{b, k_{0}}$ up to the automorphism $s: H \rightarrow H$, where $H:=\operatorname{gr}\left(Y \cup \bigcup_{b \in \Lambda\left(k_{0}\right), k_{0} \in \mathbf{N} \cup \omega_{0}} S_{b}\right)$ (see also [25]).

The family $\Xi$ order with the help of the relation $\left(H_{1}, s_{1}\right) \leq\left(H_{2}, s_{2}\right)$, if $H_{1} \subset H_{2}$ and $\left.s_{2}\right|_{H_{1}}=s_{1}$, that supplies $\Xi$ with the structure of the directed set. Every linearly ordered subset $\mathcal{C}=\left\{\left(H_{\beta}, s_{\beta}\right): \beta \in \Lambda(\mathcal{C})\right\}$ in $\Xi$ has an element $(H, s) \in \Xi$ such that $\left(H_{\beta}, s_{\beta}\right) \leq(H, s)$ for each $\left(H_{\beta}, s_{\beta}\right) \in \mathcal{C}$, where $H=\bigcup_{\beta \in \Lambda(\mathcal{C})} H_{\beta}, H \subset G,\left.s\right|_{H_{\beta}}=s_{\beta}$ for each $\beta \in \Lambda(\mathcal{C})$, here $\Lambda(\mathcal{C}) \subset \Lambda$. In view of the Kuratowski-Zorn lemma in $\Xi$ there exists a maximal element [25]. Then it need to be $(G, f)$, since each automorphism $s$ from a subgroup has an extension up to an automorphism $f$ of the entire group [16, 19]. In view of Lemma 12 the automorphism $f$ is $\left(\mathcal{A}_{\mu}, \mathcal{B}(G)\right)$-non-measurable. Since there exists not less than $2^{c}$ different bijective surjective mappings $\phi: \Lambda \rightarrow \Lambda$, then $\operatorname{card}(\Upsilon) \geq 2^{\mathrm{c}}$.

21. Remark. If $G$ is a Lie group over the field $\mathbf{K}, T_{e} G$ is the Banach space of separable type over the field $\mathbf{K}$, then measures $\mu$ on $G$ and $\nu$ on $T_{e} G$ with the needed properties from $\S 19$ exist (see [6, 10]). If $G$ is a $C^{\infty}$ over $\mathbf{R}$ or $C^{\omega}$ over a local non-archimedean field Banach-Lie group, then $G$ as the manifold has the $C^{\infty}$ exponential mapping (see [4, 5, 12]).

\section{Non-measurable automorphisms of groups relative to measures with values in local fields}

To avoid misunderstandings we first remind the basic definitions.

1. Definitions. Let $X$ be a completely regular totally disconnected topological space, let also $\mathcal{R}$ be its covering ring of subsets in $X, \bigcup\{A: A \in \mathcal{R}\}=X$. We call the ring separating, if for each two distinct points $x, y \in X$ there exists $A \in \mathcal{R}$ such that $x \in A$, $y \notin A$. A subfamily $\mathcal{S} \subset \mathcal{R}$ is called shrinking, if an intersection of each two elements from $\mathcal{A}$ contains an element from $\mathcal{A}$. If $\mathcal{A}$ is a shrinking family, $f: \mathcal{R} \rightarrow \mathbf{K}$, where $\mathbf{K}=\mathbf{R}$ or $\mathbf{K}$ is the field with the non-archimedean norm, then it is written $\lim _{A \in \mathcal{S}} f(A)=0$, if for each $\epsilon>0$ there exists $A_{0} \in \mathcal{S}$ such that $|f(A)|<\epsilon$ for each $A \in \mathcal{S}$ with $A \subset A_{0}$.

A measure $\mu: \mathcal{R} \rightarrow \mathbf{K}$ is a mapping with values in the field $\mathbf{K}$ of zero characteristic with the non-archimedean norm satisfying the following properties:

(i) $\mu$ is additive;

(ii) for each $A \in \mathcal{R}$ the set $\{\mu(B): B \in \mathcal{R}, A \subset B\}$ is bounded; 
(iii) if $\mathcal{S}$ is the shrinking family in $\mathcal{R}$ and $\bigcap_{A \in \mathcal{S}} A=\emptyset$, then $\lim _{A \in \mathcal{S}} \mu(A)=0$.

Measures on $\operatorname{Bco}(X)$ are called tight measure, where $\operatorname{Bco}(X)$ is the ring of clopen (simultaneously open and closed) subsets in $X$.

For each $A \in \mathcal{R}$ there is defined the norm: $\|A\|_{\mu}:=\sup \{|\mu(B)|: B \subset A, B \in \mathcal{R}\}$. For functions $f: X \rightarrow \mathbf{K}$ and $\xi: X \rightarrow[0,+\infty)$ define the norm $\|f\|_{\xi}:=\sup \{|f(x)| \xi(x): x \in X\}$. Put also $N_{\mu}(x):=\inf \left\{\|U\|_{\mu}: x \in U \in \mathcal{R}\right\}$. If a function $f$ is a finite linear combination over the field $\mathbf{K}$ of characteristic functions $\chi_{A}$ of subsets $A \subset X$ from $\mathcal{R}$, then it is called simple. A function $f: X \rightarrow \mathbf{K}$ is called $\mu$-integrable, if there exists a sequence $f_{1}, f_{2}, \ldots$ of simple functions such that there exists $\lim _{n \rightarrow \infty}\left\|f-f_{n}\right\|_{N_{\mu}}=0$.

The space $L(\mu)=L(X, \mathcal{R}, \mu, \mathbf{K})$ of all $\mu$-integrable functions is $\mathbf{K}$-linear. At the same time $\int_{X} \sum_{j=1}^{n} a_{j} \chi_{A_{j}}(x) \mu(d x):=\sum_{j=1}^{n} a_{j} \mu\left(A_{j}\right)$ for simple functions extends onto $L(\mu)$, where $a_{j} \in \mathbf{K}, A_{j} \in \mathcal{R}$ for each $j$.

Put $\mathcal{R}_{\mu}:=\left\{A: A \subset X, \chi_{A} \in L(\mu)\right\}$. For $A \in \mathcal{R}_{\mu}$ let $\bar{\mu}(A):=\int_{X} \chi_{A}(x) \mu(d x)$.

An automorphism $\phi$ of a totally disconnected Hausdorff topological group $G$ is called $\mu$-non-measurable, if it is $\left(\mathcal{R}_{\mu}, \mathcal{R}\right)$-non-measurable.

A totally disconnected compact Hausdorff group $G$ is called $p$-free, if it does not contain any open normal subgroup of an index divisible by $p$.

Let $G$ be a totally disconnected Hausdorff locally compact group, let also $\mathrm{B}_{\mathrm{c}}(G)$ be a covering ring of clopen compact subsets in $G$, suppose that $\mu: \mathrm{B}_{\mathrm{c}}(G) \rightarrow \mathbf{K}$ is a finitelyadditive function such that its restriction $\left.\mu\right|_{A}$ for each $A \in \mathrm{B}_{\mathrm{c}}(G)$ is a tight measure. A measure $\mu: \mathrm{B}_{\mathrm{c}}(G) \rightarrow \mathbf{K}$ is called left- (right-)invariant Haar measure, if $\mu(g A)=\mu(A)$ $\left(\mu(A g)=\mu(A)\right.$ respectively) for each $A \in \mathrm{B}_{\mathrm{c}}(G)$ and $g \in G$.

A measure $\eta: \mathcal{R} \rightarrow \mathbf{K}$ is called absolutely continuous relative to a measure $\mu: \mathcal{R} \rightarrow \mathbf{K}$, if there exists a function $f \in L(\mu)$ such that $\eta(A)=\int_{X} \chi_{A}(x) f(x) \mu(d x)$ for each $A \in \mathcal{R}$, denote it by $\eta \preceq \mu$. If $\eta \preceq \mu$ and $\mu \preceq \eta$, then we say that $\eta$ and $\mu$ are equivalent $\eta \sim \mu$.

2. Lemma. Let $X$ be a Tychonoff (completely regular) totally disconnected dense in itself topological space with a tight measure $\mu$ such that for each $x \in X$ the function $N_{\mu}(x)>0$ is non-negative, moreover, $\mu$ has not atoms in $X$. If $f: X \rightarrow X$ is a bijective epimorphic mapping such that $\operatorname{card}(f(U) \cap V) \geq \mathrm{c}:=\operatorname{card}(\mathbf{R})$ for each open subsets $U$ and $V$ in $X$, then $f$ and $f^{-1}$ are not $\left(\mathcal{R}_{\mu}, \operatorname{Bco}(X)\right)$-measurable.

Proof. Since $f$ is the bijective mapping from $X$ onto $X$, then $f^{-1}(f(U) \cap V)=U \cap$ $f^{-1}(V)$, consequently, $\operatorname{card}\left(U \cap f^{-1}(V) \geq \mathrm{c}\right.$ for each $U$ and $V$ open in $X$. In view of Lemma 7.2 [20] $\left\|\chi_{U}\right\|_{N_{\mu}}=\|U\|_{\mu}$ for each $U \in \mathcal{R}$. Due to Lemma 7.5 [20] $N_{\mu}(x)=N_{\bar{\mu}}(x)$ for each $x \in X, L(\mu)=L(\bar{\mu})$ and $\mathcal{R}_{\bar{\mu}}=\mathcal{R}_{\mu}$. Theorem 7.6 [20] states that $N_{\mu}(x)$ is upper semicontinuous and for each $\epsilon>0$ the set $\left\{x \in A: N_{\mu}(x) \geq \epsilon\right\}$ is $R_{\mu}$-compact. If $x_{0} \in X$, then $0 \leq N_{\mu}\left(x_{0}\right)<\infty$, and for each $x_{0} \in X$ and $r>N_{\mu}\left(x_{0}\right)$ there exists a neighborhood $P$ of the point $x_{0}$ such that for each point $x \in P$ there is accomplished the inequality $N_{\mu}(x)<r$. Then for each $\epsilon>0$ there exists an open subset $W$ in $X$ such that $A \subset W$ and $N_{\mu}(x)<\epsilon$ for every $x \in W \backslash A$.

For arbitrary clopen subsets $U$ and $V$ of finite $\mu$ measure take clopen subsets $U_{1}$ and $U_{2}$ in $U, V_{1}$ and $V_{2}$ in $V$ such that $0<\|U\|_{\mu}<\infty, 0<\|V\|_{\mu}<\infty, U_{1} \cap U_{2}=\emptyset, V_{1} \cap V_{2}=\emptyset$, $0<\delta \leq \min \left(\left\|U_{1}\right\|_{\mu},\left\|U_{2}\right\|_{\mu},\left\|V_{1}\right\|_{\mu},\left\|V_{2}\right\|_{\mu}\right) / s,\left\|U \backslash\left(U_{1} \cup U_{2}\right)\right\|_{\mu} \leq \delta / s,\left\|V \backslash\left(V_{1} \cup V_{2}\right)\right\|_{\mu} \leq \delta / s$. This is possible, since $N_{\mu}(x)>0$ for each $x \in X$, while $\mu$ has not atoms.

Denote $A:=f^{-1}(U), A_{j}:=f^{-1}\left(U_{j}\right)$. By the supposition of this Lemma $\operatorname{card}\left(A_{j} \cap V_{k}\right) \geq \mathrm{c}$ for each $j, k \in\{1,2\}$. Suppose that $f$ is the $\left(\mathcal{R}_{\mu}, \operatorname{Bco}(X)\right)$ measurable mapping. Then there would be $A_{j} \in L(\mu)$ for $j=1,2$ and there would be open subsets $B_{j}$ in $X$ such that $A_{j} \subset B_{j}$ and $N_{\mu}(x)<\delta / s$ for each $x \in B_{j} \backslash A_{j}$ and $j=1,2$. But $A_{1} \cap A_{2}=\emptyset$, consequently, $\mu(A)=\mu\left(A_{1}\right)+\mu\left(A_{2}\right)$.

Since $N_{\mu}(x)>0$ for each $x \in X$, then there can be chosen clopen $U, U_{1}$ and $U_{2}$ such that 
$\left.N_{\mu}\right|_{\left(A_{j} \cap V\right)}>0$, where $U_{1} \cup U_{2} \subset U$. But $\operatorname{card}\left(A_{j} \cap P_{k}\right) \geq \mathrm{c}$ for each $P_{k}$ open subset in $V_{k}$. Consequently, there exists a countable sequence of open subsets $Y_{n}$ in $X$ with $Y_{n} \supset\left(A_{j} \cap V_{k}\right)$ such that $\lim _{n \rightarrow \infty} \sup \left\{N_{\mu}(x): x \in Y_{n} \backslash\left(A_{j} \cap V_{k}\right)\right\}=0$ for given $j, k, Y_{n}=Y_{n, j, k}, j, k \in\{1,2\}$. Let $C_{n}=\bigcap_{s=1}^{n} Y_{s}$, then $\left(A_{j} \cap V_{k}\right) \subset C_{n+l} \subset C_{n}$ for any $n, l \in \mathbf{N}$, every $C_{n}$ is open in $X$, $C_{n}=C_{n, j, k}$.

At the same time $\lim _{n \rightarrow \infty} \sup \left\{N_{\mu}(x): x \in C_{n} \backslash\left(A_{j} \cap V_{k}\right)\right\}=0$. Since $\operatorname{card}\left(A_{j} \cap P\right) \geq \mathrm{c}$ for each subset $P$ open in $X$, then for each $\epsilon>0$ there exists $n_{0} \in \mathbf{N}$ such that $\operatorname{Int}(\{x \in$ $\left.\left.C_{n} \backslash C_{n+l}: N_{\mu}(x) \geq \epsilon\right\}\right)=\emptyset$ for each $n>n_{0}$ and $l \in \mathbf{N}$, where $\operatorname{Int}(B)$ denotes the interior of the subset $B$ in $X$. Then $\mu\left(A_{j} \cap V_{k}\right)=\bar{\mu}\left(c l_{X}\left(A_{j} \cap V_{k}\right)\right)=\mu\left(c l_{X}\left(A_{j} \cap V_{k}\right)\right)=\mu\left(V_{k}\right)$, since $c l_{X}\left(A_{j} \cap V_{k}\right)=V_{k} \in \mathcal{R}_{\mu}(X)$, where $X$ is the completely regular space dense in itself, here $c l_{X}(B)$ denotes the closure of a subset $B$ in $X$.

But then it would be $\sum_{j=1}^{2} \mu\left(A_{j} \cap V_{k}\right)=2 \mu\left(V_{k}\right)$, that contradicts to the additivity of the measure: $\left.2 \mu\left(V_{k}\right)=\mu\left(A_{1} \cap V_{k}\right)+\mu\left(A_{2} \cap V_{k}\right)=\mu\left(\left(A_{1} \cup A_{2}\right) \cap V_{k}\right)=\bar{\mu}\left(c l_{X}\left(A_{1} \cup A_{2}\right) \cap V_{k}\right)\right)=$ $\left.\mu\left(c_{X}\left(A_{1} \cup A_{2}\right) \cap V_{k}\right)\right)=\mu\left(V_{k}\right)$, since the characteristic of the field $\mathbf{K}$ is zero, $\operatorname{char}(\mathbf{K})=0$. Consequently, $f$ is not measurable (see also Theorem 7.12 [20]).

Applying this proof to $f^{-1}$ instead of $f$ we get that $f^{-1}$ as well is $\mu$-non-measurable, since $f^{-1}$ satisfies conditions of the second section of the proof.

3. Theorem. Let $G$ be a non-trivial locally compact Lie group over the non-archimedean local field $\mathbf{F}, \mathbf{F} \supset \mathbf{Q}_{\mathbf{p}}$, and $\mu$ be a non-trivial tight Haar measure on $G$ with values in a local field $\mathbf{K} \supset \mathbf{Q}_{\mathbf{s}}$, where $p$ and $s$ are mutually prime numbers, $(p, s)=1$. Then the group of its automorphisms Aut $(G)$ has a family of the cardinality not less than $2^{c}$ of distinct $\mu$-non-measurable automorphisms on $G$, where $\mathrm{c}:=\operatorname{card}(\mathbf{R})$ denotes the cardinality of the continuum.

Proof. Since the group $G$ belongs to the class of smoothness $C^{\omega}$, then for it the Lie algebra $\mathbf{g}$ over $\mathbf{F}$ is defined. This Lie algebra is the finite-dimensional space over $\mathbf{F}, \operatorname{dim}_{\mathbf{F}} \mathbf{g}=$ $n \in \mathbf{N}$. Then the additive group for $\mathrm{g}$ is $s$-free. In view of the Monna-Springer theorem 8.4 [20] a Haar measure $\nu^{n}$ on it is defined with values in $\mathbf{K}$ such that $\nu^{n}\left(B\left(\mathbf{F}^{\mathbf{n}}, 0,1\right)\right)=1$. It is known, that the Haar measure on $\mathrm{B}_{\mathrm{c}}(G)$ has not atoms.

Take a clopen compact subgroup $W$ in $G$ and an automorphism $\phi$ of the group $G$ from $\S 2.13$. Without loss of generality choose $W$ such that for it there is satisfied the Campbell-Hausdorff formula. In view of Lemma 2 of this section the automorphism $\phi$ is not $\mu$-measurable.

4. Corollary. The family $\Upsilon$ of non-measurable automorphisms from Theorem 3 has a subfamily $\Omega$ of the cardinality card $(\Omega) \geq 2^{c}$ such that every $f \in \Omega$ being restricted on any one-parameter subgroup over the field $\mathbf{F}$ in $G$ is non-measurable relative to the corresponding Haar measure on the subgroup with values in $\mathbf{K}$.

Proof. The exponential mapping exp from a neighborhood of zero $V_{0}$ in the algebra $\mathrm{g}$ onto a neighborhood $U_{e}$ of the unit element in $G$ induces the image of the measure $\nu_{\exp }^{n}$ on $U_{e}$, where $n$ is the dimension of $\mathrm{g}$ as the linear space over the field $\mathbf{F}, \nu^{n}$ is the Haar measure on $\mathbf{F}^{\mathbf{n}}$ as the additive group, moreover, it has not atoms.

Theorem 7.34 in [20] states that if there are two measures $\lambda$ and $\zeta$ on a covering ring $\mathcal{R}$ of a topological completely regular totally disconnected space $X$, then the following two conditions are equivalent: $(\alpha)$ there exists a locally $\zeta$-integrable function $h$ such that $\lambda(d x)=$ $h(x) \zeta(d x) ;(\beta)$ for each $x \in X$ there exists $b \in \mathbf{K}$ with $N_{\lambda-b \zeta}(x)=0$. Consequently, the measure $\nu_{\exp }^{n}$ is equivalent to the restriction of the Haar measure $\mu$ on $U_{e}$, since exp is the locally bijective of class $C^{\omega}$ mapping. Then the Haar measure $\nu$ on $\mathbf{F}$ also induces the measure $\eta_{g}$ on the one-parameter subgroup $g_{W}=\left\{g^{t}: t \in \mathbf{F},|t|<\epsilon\right\}, 0<\epsilon$, where $g \in g_{W}$. This measure $\eta_{g}$ is equivalent to the Haar measure $\mu_{g}$ on $g_{W}$.

Then from $\S \S 2.13,3.3$ and Lemma 3.2 the statement of this corollary follows. 
5. Theorem. Let $\mathrm{g}$ be a non-trivial Lie algebra finite-dimensional over the field $\mathbf{F}$ with a measure $\mu$ equal to the non-trivial $\mathbf{K}$-valued Haar measure on an additive group of $\mathbf{g}$. Then the algebra $\mathrm{g}$ has the family of the cardinality $2^{\mathbf{c}}$ of $\mu$-non-measurable automorphisms.

Proof. Take any algebraic automorphism $\phi$ of the field $\mathbf{F}$ from the proof of Theorem 2.13. Since $\mathrm{g}$ is finite-dimensional over the field $\mathbf{F}$, then the non-trivial Haar measure $\mu$ on $g$ as the additive group for $\mathbf{g}$ with values in $\mathbf{K}$ is equivalent to the measure $\nu^{n}$ (see Definitions 1). The automorphism $\phi$ extends up to an automorphism of the algebra: $\phi\left(a_{j} v_{j}\right)=\phi\left(a_{j}\right) v_{j}$, $\phi\left(a_{1} v_{1}+\ldots+a_{m} v_{m}\right)=\phi\left(a_{1}\right) v_{1}+\ldots+\phi\left(a_{m}\right) v_{m}, \phi\left(\left[a_{k} v_{k}, a_{j} v_{j}\right]\right)=\left[\phi\left(a_{k}\right) v_{k}, \phi\left(a_{j}\right) v_{j}\right]$ for each $a_{j} \in \mathbf{F}, k, j=1, \ldots, m$, where $v_{1}, \ldots, v_{m}$ is the basis of generators in $\mathrm{g}$ (see Definitions 2.1). Therefore, due to Lemma 2 the automorphism $\phi$ of the algebra $g$ is $\left(B_{c \mu}, B_{c}(g)\right)$-nonmeasurable. The family of such different automorphisms of the algebra $g$ has the cardinality $2^{c}$.

6. Let $G$ be a $C^{\omega}$ non-trivial Lie group over a non-archimedean local field $\mathbf{F}$, moreover, $G$ be complete as an uniform space. Suppose that $\mu: \mathcal{R}(G) \rightarrow \mathbf{K}$ is finitely-additive and there exists a clopen subgroup $W$ in $G$ such that the restriction $\left.\mu\right|_{W}$ is the tight non-trivial measure on $\operatorname{Bco}(W)$ with values in $\mathbf{K}$ such that $N_{\mu}(g)>0$ for each $g \in G$, moreover, $\mu$ has not any atoms, where $\operatorname{Bco}(G) \supset \mathcal{R}(G) \supset \operatorname{Bco}(W), \mathcal{R}(G)$ is a covering ring for $G$. Let $\exp : V \rightarrow W$ be the exponential mapping for $G$ as the analytic $C^{\omega}$ manifold over $\mathbf{F}$ from an open neighborhood $V$ of zero in $T_{e} G$ on $W$. Also suppose that a tight $\mathbf{K}$-valued measure $\nu$ on $\mathcal{R}\left(T_{e} G\right)$ is such that $\nu(J)=\mu(\exp (J))$ for each $J \in \operatorname{Bco}(V)$, where $T_{e} G$ is the linear space of separable type over the field $\mathbf{F}, \operatorname{Bco}(\mathrm{g}) \supset \mathcal{R}(\mathrm{g}) \supset \operatorname{Bco}(V), \mathcal{R}(\mathrm{g})$ is the covering ring for $\mathrm{g}$. Let as in Theorem $3 p$ and $s$ be two mutually prime numbers, $(p, s)=1$.

Suppose that $G$ has a clopen subgroup $W$, exp : $V \rightarrow W$, moreover, in $W$ there exists an everywhere dense subgroup $S$ such that the restriction $\left.\ln \right|_{S}$ corresponds to the CampbellHausdorff formula, where $\ln$ is the inverse mapping to exp.

Let $\pi_{v}: T_{e} G \rightarrow \mathbf{F} v$ be a $\mathbf{F}$-linear projection operator, where $\nu_{v}$ is equivalent to the Haar measure on $\mathbf{F}$, where $v$ is the non-zero vector of the tangent space $v \in T_{e} G, \nu_{v}(J)=\nu\left(\pi_{v}^{-1}(J)\right)$ for each $J \in \mathrm{B}_{\mathrm{c}}(\mathbf{F})$.

Theorem. Then such group $G$ has a family $\Upsilon$ of $\left(\mathcal{R}(G)_{\mu}, \mathcal{R}(G)\right)$-non-measurable automorphisms of the cardinality not less than $2^{\mathrm{c}}$, $\operatorname{card}(\Upsilon) \geq 2^{\mathrm{c}}$. Moreover, $\Upsilon$ has a subfamily $\mathcal{P}$ of automorphisms $f$, restrictions of which on one-parameter over $\mathbf{F}$ local subgroups $\{\exp (x v):|x|<\epsilon\}$ in $S$ are non-measurable relative to the corresponding $\mathbf{K}$-valued Haar measure on $\{\exp (x v):|x|<\epsilon\}$.

Proof. The image $\nu$ on $V$ of the measure $\mu$ with the help of the mapping $\ln , \nu(B)=$ $\mu(\exp (B))$ for every $B \in \mathrm{Bco}(V)$ is extendable up to the tight measure on the corresponding covering ring $\mathcal{R}(\mathrm{g}), \nu(B):=\sum_{j=1}^{\infty} \nu\left(\left(B-h_{j}\right) \cap V\right) s^{j}$ for every $B \in \mathcal{R}(\mathrm{g})$, where $\left\{\left(V+h_{j}\right)\right.$ : $\left.j \in \mathbf{N}, h_{j} \in \mathrm{g}\right\}$ is the covering for $\mathrm{g}$, since $\mathbf{g}$ by the condition has the separable type over $\mathbf{F}$, while the field $\mathbf{F}$ is separable and locally compact. As $\mathcal{R}(\mathrm{g})$ we can take the minimal ring generated by $\bigcup_{j=1}^{\infty} \operatorname{Bco}\left(V+h_{j}\right)$. Therefore, $\nu$ has tight measures as the projections $\nu_{\omega\left(\mathbf{F}^{\mathbf{n}}\right)}$ on $\omega\left(\mathbf{F}^{\mathbf{n}}\right)$ for each embedding $\omega: \mathbf{F}^{\mathbf{n}} \hookrightarrow \mathrm{g}$ as the $\mathbf{F}$-linear space, $\nu_{\omega\left(\mathbf{F}^{\mathbf{n}}\right)}(B)=\nu\left(\pi^{-1}(B)\right)$ for each $B \in \mathbf{B}_{c}\left(\omega\left(\mathbf{F}^{\mathbf{n}}\right)\right)$, where $\pi: \mathrm{g} \rightarrow \omega\left(\mathbf{F}^{\mathbf{n}}\right)$ is the projection operator.

The operator $\pi$ is $\mathbf{F}$-linear and it exists, since $\omega\left(\mathbf{F}^{\mathbf{n}}\right)$ is finite-dimensional over $\mathbf{F}$, while the field $\mathbf{F}$ is locally compact (see Theorems 5.13 and 5.16 [20] and [15]). The image $\left.\nu_{\omega(\mathbf{F})}\right|_{V \cap \omega(\mathbf{F})}$ with the help of exp generates the measure on the local one-parameter subgroup in $S$, $\mu_{g}(B)=\nu_{\omega(\mathbf{K})}(\ln (B))$ for every $B \in \mathcal{R}\left(g_{W} \cap W\right)$, which extends up to a tight measure $\mu_{g}$ on $g_{W}$.

Take an automorphism $f$ of the group $G$ from $\S 2.19$. In view of Lemma 3.2 and $N_{\mu}(g)>0$ for each $g \in G$ we get that $f$ is $\mu$-non-measurable. The non-measurability of restrictions 
of $f$ on one-parameter subgroups relative to the Haar measures on them follows from the properties of $\phi$ again due to Lemma 3.2 and $\S 2.13$. The families $\Upsilon$ and $\mathcal{P}$ of such different automorphisms of the group $G$ due to $\$ 2.19$ have the cardinalities not less than $2^{c}$.

7. Theorem. Let $G$ be an infinite topological totally disconnected dense in itself Hausdorff group with a non-trivial tight measure $\mu$ on $G$ having no any atom, moreover, $N_{\mu}(g)>0$ for each $g \in G$, while $G$ is complete as the uniform space, card $(U) \geq$ c for each open $U$ in $G$. Then there exists a family $\Upsilon$ of $\mu$-non-measurable distinct automorphisms of the group $G$ of the cardinality $\operatorname{card}(\Upsilon) \geq 2^{c}$.

Proof. Take an automorphism $\phi$ of the group $G$ from $\S 2.20$. In view of Lemma 3.2 it is $\mu$-non-measurable. The family of such distinct automorphisms of the group $G$ due to $\S 2.20$ has the cardinality not less than $\operatorname{card}(\Upsilon) \geq 2^{\mathrm{c}}$.

8. Remark. If $G$ is a Lie group over the field $\mathbf{F}$ of the class $C^{\omega}$, its tangent space $T_{e} G$ is a Banach space of separable type over the field $\mathbf{F}$, then there exist measures $\mu$ on $G$ and $\nu$ on $T_{e} G$ with the desired properties from $\S 3.6$ (see [11]).

\section{References}

[1] Bachturin Yu. Basic structures of modern algebra. Moscow, Nauka, 1990.

[2] Bichteler K. Inventiones Mathem.// On the existence of noncontinuous irreducible representations of locally compact group. 1968. V. 6 (2). P. 159-162.

[3] Bourbaki N. Integration. Vector integration. Haar measure. Convolution and representations. Moscow, Nauka, 1970.

[4] Bourbaki N. Lie groups and algebras. Chapters 1-8. Moscow, Nauka, 1972-78.

[5] Bourbaki N. Differentiable and analytic manifolds. Moscow, Mir, 1975.

[6] Dalecky Yu.L., Fomin S.V. Measures and differential equations in infinite dimensional space. Dordrecht, Kluwer, 1991.

[7] Ludkovsky S.V. Classification of some types of locally compact groups by their unitary representations// Usp. Mathem. Nauk 1992. V. 47. N 5. P. 185-186.

[8] Ludkovsky S.V. Measurability of representations of locally compact groups// Mathem. Sborn. 1995. V. 186. N 2. P. 83-92.

[9] Ludkovsky S.V. Measurability of automorphisms of topological groups// Mathem. Zametki 2000. V. 68. N 1. P. 105-112.

[10] Ludkovsky S.V. Quasi-invariant and pseudo-differentiable real-valued measures on a non-archimedean Banach space // Analysis Mathem. 2002. V. 28. P. 287-316 (previous variants: Preprint ICTP N IC/96/210, October 1996, 50 p.; Los Alamos preprint math.GM/0106169).

[11] Ludkovsky S.V. Quasi-invariant and pseudo-differentiable measures on nonArchimedean Banach spaces with values in non-Archimedean fields // J. Math. Sci. 2004. V. 122: 1. P. 2949-2983 (previous variant: Los Alamos preprint math.GM/0106170).

[12] Klingenberg W. Riemannian geometry. N.Y., Walter de Gruyter, 1982. 
[13] Moore R.T. Measurable, continuous and smooth vectors for semigroups and group representations// Memoirs Amer. Math. Soc. 1968. V. 78. P. 1-80.

[14] Naimark M.A. Normed rings. Moscow, Nauka, 1968.

[15] Narici L., Beckenstein E. Topological vector spaces. New York, Marcel Dekker Inc., 1985.

[16] Neumann B.H., Neumann H. Extending partial endomorphisms of groups// Proc. Lond. Math. Soc. 1952. V. (3) 2. P. 337-348.

[17] Plotkin B.I. Groups of automorphisms of algebraic systems. Moscow, Nauka, 1966.

[18] Pontryagin L.S. Continuous groups. Moscow, Nauka, 1984.

[19] Puusemp P. Endomorphisms and endomorphism semigroups of groups. N.Y., Nova Science Publishers Inc., 2006. P. 27-57 (in: Focus on group theory research. Ed.: L.M. Ying).

[20] Rooij A.C.M. van. Non-archimedean functional analysis. New York, Marcel Dekker Inc., 1978.

[21] Fell J.M.G., Doran R.S. Representations of *-algebras, locally compact groups, and Banach *-algebraic bundles. Boston, Academic Press, 1988.

[22] Federer G. Geometric measure theory. Moscow, Nauka, 1987.

[23] Hewitt E., Ross K.A. Abstract harmonic analysis. Moscow, Nauka, 1975.

[24] Schikhof W.H. A Radon-Nykodim theorem for non-archimedean integrals and absolutely continuous measures on groups // Indag. Math. Ser. A. 1971. V. 33: 1. P. 78-85.

[25] Engelking R. General topology. Moscow, Mir, 1986.

[26] Weil A. Basic number theory. Berlin, Springer-Verlag, 1973. 